Brazilian Journal

of Chemical

ISSN 0104-6632

Engineering

Printed in Brazil

www.scielo.br/bjce

Vol. 34, No. 04, pp. 1083 - 1119, October - December, 2017

$($ (c) $)$ EY

dx.doi.org/10.1590/0104-6632.20170344s20150748

\title{
A NOVEL TRANSITION IDENTIFICATION MECHANISM FOR THE DIESEL BLENDING AND DISTRIBUTION SCHEDULING PROBLEM USING THE DISCRETE TIME REPRESENTATION WITH TWO TIME-SCALES GRANULARITY
}

\author{
D. Dimas ${ }^{1}$, V. V. Murata ${ }^{1}$ and S. M. S. Neiro ${ }^{1, *}$ \\ ${ }^{1}$ Federal University of Uberlândia, School of Chemical Engineering. Uberlândia, MG, Brazil. \\ *E-mail: srgneiro@ufu.br
}

(Submitted: November 18, 2015; Revised: April 19, 2016; Accepted: May 13, 2016)

\begin{abstract}
Transitions between tasks arise in many different scheduling problems. Sometimes transitions are undesired because they incur costs; sometimes they are undesired because they require setup time, and sometimes both. In one way or the other, frequently, transitions need to be identified and penalized in order for their frequency to be minimized. The present work is concerned with the study of alternative optimization formulations to address transitions with the blending and distribution scheduling of oil derivatives. Our study starts by revisiting a model proposed in the literature that was built considering a very short time horizon $(24 \mathrm{~h})$. Next, improvements concerning the transition constraints are evaluated and a new approach is proposed with the purpose of extending model applicability to cases where longer time horizons are of interest. The new proposed mechanism of evaluating transitions relies on aggregating the detailed discrete time scale (hours) to a higher and less detailed level (days). Transitions are then evaluated on the lower level of aggregation with the benefit of reducing the number of required constraints. It must also be emphasized that the proposed model is built on the basis of a set of heuristics that have direct impact on solution and solution time. Results attained for a four-day time horizon demonstrate cost savings on the order of $32 \%$ when compared with four sequenced schedules of a one-day time horizon each. Savings are mainly obtained as a consequence of the reduction of the predicted number of transitions.
\end{abstract}

Keywords: diesel blending, distribution scheduling, refinery, discrete time representation, event points.

\section{INTRODUCTION}

In the oil industry the growing demand for petroleum derivatives, stringent environmental regulations and increased market competition have driven the companies to improve operations management, reduce cost and operate more efficiently. In such an aggressive environment, optimization of the plan and schedule is a valuable differentiator that creates nontrivial cost reduction opportunities. Historically, oil industry scheduling has normally been considered for subsystems of the refinery due to the high degree of complexity involved in addressing the problem globally, although efforts treating more than one subsystem of the refinery simultaneously can be identified (Gothe-Lundgren et al., 2002, Simão et al., 2007; Luo and Rong, 2007; Shah et al., 2009,

\footnotetext{
* To whom correspondence should be addressed
} 
Harjunkoski et al., 2014, Sha and Ierapetritou, 2015; Gao et al., 2015). Jia and Ierapetritou (2004) decompose the refinery scheduling problem into three subsystems: (i) crude oil unloading, mixing and inventory control; (ii) production unit scheduling, and (iii) product blending and distribution. In the last two decades, a lot of attention has been devoted to studying the crude oil supply operation since it is an important activity that can affect directly the entire refinery operation. The reported literature has comprised a gamut of subjects, covering aspects related to problem features (different topologies, restrictive operating rules, and volume decisions), time representation, model tightness and solution strategies (Shah, 1996; Pinto et al., 2000; Jia et al., 2003; Moro and Pinto, 2004; Furman et al. 2007; Saharidis and Ierapetritou, 2009; Mouret et al. 2011; Chen et al. 2012).

On the distribution side, the main concern is how to distribute large volumes of products with the most cost effective schedule. The most common and reliable mode of transportation used in the oil industry is the pipeline. According to Rejowski and Pinto (2003), pipelines are also used to distribute oil derivatives due to their capability to transport several products for long distances with lower cost. Rejowski and Pinto (2003) proposed an optimization model for a system composed of one refinery, a single pipeline and five depots disposed along the pipeline, whose objective was to minimize the distribution cost composed of inventory, pumping and transition costs. The model was based on disjunctive programming and discrete time formulation. In a subsequent work, Rejowski and Pinto (2004), introduced a set of integer cuts and special interface constraints to their previous model resulting in better solutions. Cafaro and Cerdá (2004) considered the same problem, proposing a model that did not rely on the discretization of both time and volume. In another vein, Relvas et al. (2007) addressed the integration of pipeline and distribution depot through an MILP model combining inventory management and pipeline sequencing operation, which was applied to a real-world case comprising a Portuguese company. Cafaro and Cerdá (2009) addressed a problem comprised of a pipeline network with multiple sources and destinations. The proposed MILP continuous model was able to determine the pipeline input streams considering different sources, batch size and pumping timing. Boschetto et al. (2010) developed a model for a Brazilian system composed of four refineries, two harbors, two market clients, six depots and thirty bidirectional pipelines. They proposed a hierarchical decomposition strategy integrating heuristics and the MILP model to solve the resulting complex problem. MirHassani and BeheshtiAsl (2013) also used heuristics to solve a MILP problem that involved a refinery, a pipeline and a distribution center. Recently, Ghaffari-Hadigheh and Mostafaei (2014) developed a mathematical model considering simultaneous deliveries to multiple terminals combining continuous representation for both volume and time. Similarly, Cafaro et al. (2015) introduced an MINLP continuous model for single source pipeline with simultaneous deliveries in several terminals in which friction loss related with the pumping cost was included.

The blending problem, also known as pooling, has been the focus of many contributions published in the scientific literature. Rigby et al. (1995) solved offlineblending problems with nonlinear recipe optimization for the Texaco Company using the GAMS system. Glismann and Gruhn (2001) did a study based on the RNT (Resource task network) representation that integrated the product scheduling and blending recipe optimization. A decomposition procedure was proposed that solves first a nonlinear problem to determine the product recipe and volumes, after which an MILP problem determines the best sequence operation. Jia and Ierapetritou (2003) proposed a simultaneous scheduling of gasoline blending and distribution based on continuous time where constant recipe has been considered to maintain the model linearity. Mendez et al. (2006) developed an MINLP model for optimizing simultaneously blending recipes and the short term scheduling considering identical blenders. Li et al. (2010) proposed a continuous time model for scheduling gasoline blending and integrating several operations. The model considers a set of features such as parallel nonidentical blenders, multiple demands, blending and storage transitions among other practical rules. Later, Li and Karimi (2011) improved that work by considering setup time for blenders and using the unit slot representation, which enabled them to achieve better solutions. More recently, Shah and Ierapetritou (2015) proposed a Lagrangian decomposition algorithm for integrating production unit scheduling with product blending and distribution. A canonical piecewise linear model (CPWL) was developed by Gao et al (2015) to approximate nonlinearities into linear pieces, which transforms the MINLP model into an MILP. The proposed model was applied to a real-world problem involving the scheduling of diesel and gasoline blender's operation from a Chinese refinery. Castillo-Castillo and Mahalec (2016) addressed a continuous time formulation for the gasoline blending scheduling based on the work by Li and Karimi (2011). They have significantly improved the former work by adding operational constraints, a procedure to reduce the number of binary variables and a way to set a lower bound to the objective function.

The present work focuses on the diesel blending and distribution scheduling problem. Pinto et al. (2000) introduced a formulation addressing this problem. However, the proposed formulation was built relying on a very short term horizon $(24 \mathrm{~h})$. In this work, extensions to the formulation proposed by Pinto and coworkers are proposed in the form of different approaches. The main target of the present work is to discuss different forms of identifying transitions in the distribution of oil products for extending the scheduling horizon. 
The text is organized as follows: in section 2 a description of the problem addressed in this work is detailed. In section 3 the base model is presented. In section 4 three approaches are presented for addressing short term horizons and another approach is proposed for addressing long term horizons. Results and discussions are presented in section 5. Finally, the concluding remarks are brought up in section 6 .

\section{PROBLEM STATEMENT}

The target problem involves a set of distillation units, which provide intermediate streams that are stored in dedicated storage tanks (Figure 1). Only cuts related to diesel production are in scope for the present problem. Taking into consideration that each distillation unit operates continuously and tanks cannot load and unload simultaneously, two run-down tanks are required for each distillation unit so that their cuts are available for composing the final products at any time along the entire scheduling horizon. The quality of the content in each of the two rundown tanks is the same as that of the cut provided by its corresponding feeding unit and considered constant for the duration of the scheduling horizon as if the refinery was developing a campaign imposed by planning, which is a reasonable assumption for short scheduling cycles. It is also assumed that the quality of the initial inventory in each of the intermediate tanks coincides with that of the underlying campaign, so that no mixing occurs at the rundown tanks.
Finished products are obtained by mixing the cuts produced by the distillation units, which are then sent through unidirectional pipelines to local markets. Each diesel grade is differentiated by two quality indicators; sulfur content and cetane number, which for simplicity are assumed to be determined as the weighted average of the volumes used of each cut. The blended products are pumped to the final destinations without going through intermediate storage tanks, i.e., blending is done in-line prior to feeding pipelines. Full connectivity between tanks and pipelines is assumed. Pipelines operate independently and under unique demand requirements. Diesel parcels with different grades are pumped contiguously, in which case an interface between two adjacent parcels establishes an undesirable interface containing off-spec material that demands appropriate handling.

The problem scope is as follows, given:

- The diesel related cuts produced by distillation units and their qualities;

- Initial intermediate material inventory;

- Tank capacity;

- Pumping capacity;

- Product demand and specifications;

- Time horizon.

Determine:

- Tank operations management and inventory profile;

- Consumption of intermediate streams for each

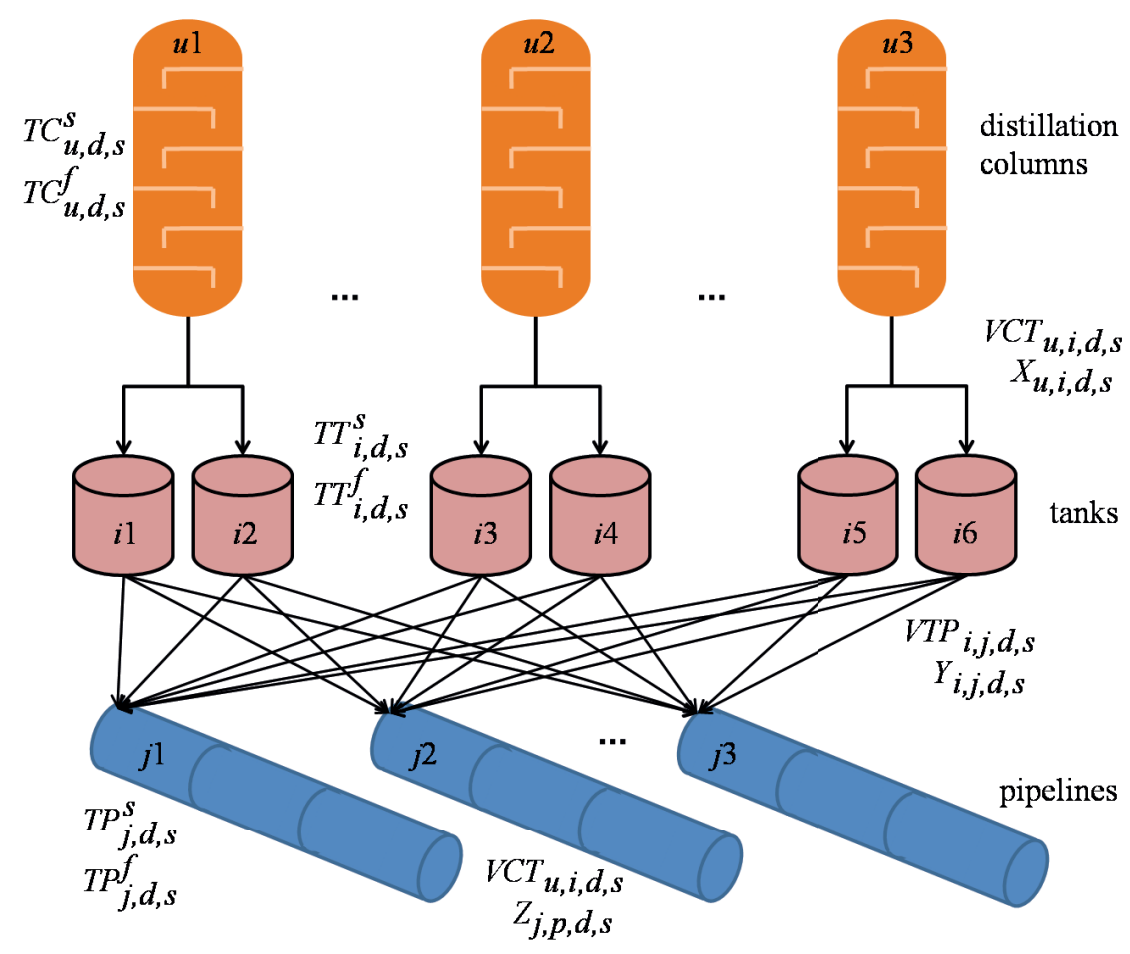

Figure 1. Schematic of blending and distribution infrastructure for diesel production. 
blended product for fulfilling demand and quality specifications;

- Pipeline operations schedule.

The objective function is to minimize costs, which include raw material, pumping, inventory costs as well as costs resulting from off-spec material generated at the interface in between blended diesel parcels transported through pipelines. The problem is subjected to the operating rules previously postulated.

\section{MATHEMATICAL FORMULATION}

Pinto et al. (2000) addressed the problem described in the problem statement and the complete model is presented next in detail with minor corrections. The mathematical model was built based on discrete time representation, which relies on a number of equally spaced time points resulting in an MILP model. The following nomenclature is used in the formulation presented in this section and in the studied approaches in the next section:

\section{Indices and Sets}

$\boldsymbol{E}_{d}$

$\boldsymbol{E}_{d}^{L}$

$\mathrm{I}_{\mathrm{j}}$

$I_{u}$

$\mathrm{J}$

$\mathrm{J}_{\mathrm{i}}$

K

$\mathrm{P}$

$\mathrm{T}$

$\boldsymbol{T}_{d}$

$\mathrm{U}$

\section{Parameters}

$\mathrm{Ci}_{\mathrm{i}}$

$\mathrm{Cp}_{\mathrm{i}}$

$\mathrm{Crm}_{\mathrm{i}}$

$\mathrm{Ct}_{\mathrm{p}, \mathrm{n}}$

$\mathrm{D}_{\mathrm{j}, \mathrm{p}}$

$\mathrm{D}_{\mathrm{j}, \mathrm{p}, \mathrm{t}}$

$\mathrm{F}^{\max }{ }_{\mathrm{i}}$

$F^{\max }{ }_{\mathrm{ij}}$

$F^{\max }{ }_{j}$

$\mathrm{F}^{\text {min }}{ }_{\text {i }}$

$\mathrm{F}^{\mathrm{min}}$

$\mathrm{F}^{\mathrm{min}}{ }_{\mathrm{j}}$

$\mathrm{H}$

set of days $(d=\{1,2, \ldots, \mathbf{D}\})$

set of events $(e=\{1,2, \ldots, \mathbf{E}\})$

set of events $e$ that belong to each day $d$

set of events $e$ that belong to each day $d$ with the exception of the last event of each day

subset of tanks $i$ that can be aligned to pipeline $j$

subset of tanks $i$ that can be loaded by distillation unit $\mathrm{u}$

set of pipelines $(j=\{1,2, \ldots, \mathbf{J}\})$

subset of pipelines $j$ that are allowed to connect to tank $i$

set of qualities $(k=\{1,2, \ldots, \mathbf{K}\})$

set of products $(n, p=\{1,2, \ldots, \mathbf{P}\})$

set of time intervals $(t=\{1,2, \ldots, \mathbf{T}\})$

set of time periods $t$ that belong to each day $d$

set of distillation $\operatorname{units}(u=\{1,2, \ldots, \mathbf{D U}\})$

inventory cost for tank $i\left(\$ / \mathrm{m}^{3}\right)$

cost associated with pumping intermediate material from tank $i$ to pipeline $\left(\$ / \mathrm{m}^{3}\right)$

raw material cost associated with using intermediate material stored in tank $i\left(\$ / \mathrm{m}^{3}\right)$

cost associated with the interface established between products $p$ and $n(\$)$

demand of end product $p$ incurred at pipeline $j\left(\mathrm{~m}^{3}\right)$

demand of product $p$ incurred at pipeline $j$ at time period $t\left(\mathrm{~m}^{3}\right)$ (usually incurred at the last time period of each day)

maximum flowrate between distillation column and tank $i\left(\mathrm{~m}^{3} / \mathrm{h}\right)$

maximum pumping flowrate between tank $i$ and pipeline $j\left(\mathrm{~m}^{3} / \mathrm{h}\right)$

maximum pumping flowrate for pipeline $j\left(\mathrm{~m}^{3} / \mathrm{h}\right)$

minimum flowrate between distillation column and tank $i\left(\mathrm{~m}^{3} / \mathrm{h}\right)$

minimum pumping flowrate between tank $i$ and pipeline $j\left(\mathrm{~m}^{3} / \mathrm{h}\right)$

minimum pumping flowrate for pipeline $j\left(\mathrm{~m}^{3} / \mathrm{h}\right)$

number of time periods $(H=|\mathbf{T}|)$

maximum number of connections between tank $i$ and pipelines

maximum number of transitions allowed at pipeline $j$

total length of time horizon (h)

initial inventory in tank $i\left(\mathrm{~m}^{3}\right)$

maximum inventory allowed in tank $i\left(\mathrm{~m}^{3}\right)$

minimum inventory allowed in tank $i\left(\mathrm{~m}^{3}\right)$

static value of quality $k$ of the intermediate material stored in tank $i$

spec-value of quality $k$ of end product $p$ 


\begin{tabular}{|c|c|}
\hline $\mathrm{ZD}_{\mathrm{j}, \mathrm{p}}$ & $0-1$ parameter that indicates if there is demand incidence for end product $p$ at pipeline $j$ \\
\hline $\mathrm{ZD}_{\mathrm{j}, \mathrm{p}, \mathrm{t}}$ & $0-1$ parameter that indicates if there is demand incidence for end product $p$ at pipeline $j$ at time period $t$ \\
\hline \multicolumn{2}{|c|}{ Continuous Variables } \\
\hline $\mathrm{F}_{\mathrm{i}, \mathrm{t}}$ & inlet flowrate of tank $i$ at time period $t\left(\mathrm{~m}^{3} / \mathrm{h}\right)$ \\
\hline $\mathrm{F}_{\mathrm{j}, \mathrm{p}, \mathrm{t}}$ & inlet flowrate of end product $p$ to pipeline $j$ at time period $t\left(\mathrm{~m}^{3} / \mathrm{h}\right)$ \\
\hline $\mathrm{F}_{\mathrm{i}, \mathrm{j}, \mathrm{t}, \mathrm{t}}$ & flowrate between tank $i$ and pipeline $j$ at time period $t\left(\mathrm{~m}^{3} / \mathrm{h}\right)$ \\
\hline $\mathrm{T}_{\mathrm{j}, \mathrm{p}, \mathrm{p}}^{\mathrm{E}}$ & end time of loading end product $p$ to pipeline $j$ \\
\hline $\mathrm{T}^{\mathrm{E}} \mathrm{j}, \mathrm{p}, \mathrm{d}$ & end time of loading end product $p$ to pipeline $j$ within day $d$ \\
\hline $\mathrm{T}_{\mathrm{j}, \mathrm{e}}$ & instant of time in which event $e$ occurs in pipeline $j$ \\
\hline $\mathrm{T}_{\mathrm{j}, \mathrm{p}}^{\mathrm{s}}$ & start time of loading end product $p$ to pipeline $j$ \\
\hline $\mathrm{T}_{\mathrm{j}, \mathrm{p}, \mathrm{d}}^{\mathrm{S}}$ & start time of loading end product $p$ to pipeline $j$ within day $d$ \\
\hline$V_{i, t}$ & inventory level at tank $i$ in time period $t\left(\mathrm{~m}^{3}\right)$ \\
\hline \multicolumn{2}{|c|}{ Binary Variables } \\
\hline $\mathrm{E}_{\mathrm{j}, \mathrm{p}, \mathrm{e}}$ & denotes if product $\mathrm{p}$ starts being pumped at event point $\mathrm{e}$ in pipeline $\mathrm{j}$ \\
\hline$P_{j, p, n}$ & indicates potential transition between product $p$ and $n$ on pipeline $j$ \\
\hline$S_{j, p, t}$ & $\begin{array}{l}\text { denotes if operation of pipeline } j \text { is interrupted at time period } t \text { and the last product loaded to the } \\
\text { pipeline was } p\end{array}$ \\
\hline $\mathrm{S}_{\mathrm{j}, \mathrm{p}, \mathrm{e}}$ & $\begin{array}{l}\text { denotes that no pumping operation is allocated to event point } \mathrm{e} \text { in pipeline } \mathrm{j} \text { and the last product loaded } \\
\text { to the pipeline was } \mathrm{p}\end{array}$ \\
\hline $\mathrm{W}_{\mathrm{j}, \mathrm{p}, \mathrm{n}}$ & denotes if product $n$ is pumped right after product $p$ in pipeline $j$ \\
\hline $\mathrm{W}_{\mathrm{j}, \mathrm{p}, \mathrm{n}, \mathrm{e}}$ & denotes if product $\mathrm{n}$ is pumped right after product pin pipeline $\mathrm{j}$ at event point $\mathrm{e}$ \\
\hline$X_{i, t}$ & denotes if $\operatorname{tank} i$ is being loaded at time period $t$ \\
\hline$Y_{i, j, t}$ & denotes if $\operatorname{tank} i$ is loading pipeline $j$ at time period $t$ \\
\hline $\mathrm{Z}_{\mathrm{j}, \mathrm{p}, \mathrm{t}}^{\mathrm{E}, \mathrm{n}}$ & denotes if end product $p$ ends being loaded to pipeline $j$ at time period $t$ \\
\hline$Z_{j, p, t, d}^{E}$ & denotes if product $\mathrm{p}$ ends being loaded to pipeline $\mathrm{j}$ at time period $\mathrm{t}$ within day $\mathrm{d}$ \\
\hline & denotes if pumping of end product $p$ to pipeline $j$ is active at time period $t$ \\
\hline$Z_{j, p, t}^{S}$ & denotes if end product $p$ starts being loaded to pipeline $j$ at time period $t$ \\
\hline & denotes if product $\mathrm{p}$ starts being loaded to pipeline $\mathrm{j}$ at time period $\mathrm{t}$ within day $\mathrm{d}$ \\
\hline
\end{tabular}

As already mentioned, the objective function is to minimize costs, which include raw material, pumping, inventory as well as transition costs resulting from the interface generated between blended diesel parcels transported through pipelines, which is mathematically stated in Equation 1.

$$
\min \sum_{i \in I} \sum_{j \in J} \sum_{t \in T}\left(C r m_{i}+C p_{i}\right) F_{i, j, t}+\sum_{i \in I} \sum_{t \in T} C i_{i} V_{i, t}+\sum_{j \in J} \sum_{p \in P} \sum_{n \in P} C t_{p, n} W_{j, p, n}
$$

The objective function is subject to the following constraints:

\section{a) Material balance constraints}

The total amount of material in each tank at each time period is given by the initial inventory plus the amounts in and out of the tank accounted since the beginning of the time horizon, as given by Equation 2. In addition, constraint 3 sets upper and lower bounds to the inventory level.

$$
V_{i, t}=V_{i}^{o}+\sum_{t^{\prime} \leq t}\left(F_{i, t^{\prime}}-\sum_{j \in J_{i}} F_{i, j, t^{\prime}}\right) \forall i \in I, t \in T
$$




$$
V_{i}^{\min } \leq V_{i, t} \leq V_{i}^{\max } \forall i \in I, t \in T
$$

Equation 4 establishes the material balance between tanks and pipelines, which sets the amount of product $p$ pumped through pipeline $\mathrm{j}$ equal to the consumed amount of raw materials. Since at a given time t only one product $\mathrm{p}$ can be loaded to the pipeline $\mathrm{j}$, the left hand side of Equation 4 has all terms null except for one.

$$
\sum_{p \in P} F_{j, p, t}=\sum_{i \in I_{j}} F_{i, j, t} \forall j \in J, t \in T
$$

Every product pumped through pipelines must satisfy quality requirements, which are guaranteed through constraints $5 \mathrm{a}$ and $5 \mathrm{~b}$. In these constraints, $x_{i, k}^{0}$ is a static parameter that represents the quality $\mathrm{k}$ of the raw material stored in storage tank $\mathrm{i}$, whereas $x_{i, k}^{s p c}$ is the specification value that the corresponding product must meet. Note that the quality calculation is a volume weighted average relationship, which can be assumed so because properties of raw materials are assumed to be constant for the duration of the scheduling horizon, which in turn means that density is also constant and thus cancelles out when put on both sides of the equations. It must also be borne in mind that the relationship between $F_{j, p, t}$ and $F_{i, j, t}$ is established through Equation 4. If the assumption of a single campaign was not assumed, Equations 5 would assume a nonlinear form, making the problem more difficult to solve. This assumption is somewhat limiting but valid for short scheduling horizons. The sign on the inequality depends on the property under consideration. Sometimes a greater than or equal sign is used and sometimes the opposite is desired. In this paper, constraint $5 \mathrm{a}$ imposes a maximum amount on the sulfur content, while constraint $5 \mathrm{~b}$ is used for imposing a minimum on the cetane number.

$$
\begin{aligned}
& \sum_{p \in P} x_{p, k}^{s p c} F_{j, p, t} \geq \sum_{i \in I} x_{i, k}^{0} F_{i, j, t} \forall j \in J, k=1, t \in T \\
& \sum_{p \in P} x_{p, k}^{s p c} F_{j, p, t} \leq \sum_{i \in I} x_{i, k}^{0} F_{i, j, t} \forall j \in J, k=2, t \in T
\end{aligned}
$$

\section{b) Demand constraint}

Besides satisfying quality specifications, the total product volume produced must meet the demand, Equation 6. Although Equation 6 imposes the condition that demand must be satisfied exactly, in the present work it will sometimes be allowed to dispatch an amount that is greater than the minimum required volume.

$$
\sum_{t \in T} F_{j, p, t}=D_{j, p} \forall j \in J, p \in P
$$

\section{c) Operating rules and logic constraints}

Since distillation columns operate continuously, intermediate products are continuously transferred to either of the available rundown tanks depending only on which tank is feeding a pipeline. At any time, however, only one rundown tank can receive the intermediate product from the distillation unit, as stated by Equation 7 .

$$
\sum_{i \in I_{u}} X_{i, t}=1 \forall u \in D U, t \in T
$$


In addition, it is forbidden for a tank to load and unload simultaneously. Therefore, at any time a tank is loading, unloading or settling. If a tank $\mathrm{i}$ is not being loaded $(\mathrm{Xi}, \mathrm{t}$ $=0$ ), then the maximum number of connections between tank $\mathrm{i}$ and pipelines $j \in J_{i}$ is given by NCi. On the other hand, if a tank $\mathrm{i}$ is being loaded $(\mathrm{Xi}, \mathrm{t}=1)$, constraint 8 forbids unloading to any pipeline by setting all $\mathrm{Yi}, \mathrm{j}, \mathrm{t}=$ 0 . Therefore, constraint 8 serves two purposes: it forbids simultaneous loading and unloading and at the same time limits the maximum number of connections between tanks and pipelines.

$$
N C_{i} X_{i, t}+\sum_{j \in J_{i}} Y_{i, j, t} \leq N C_{i} \forall i \in I, t \in T
$$

Pinto et al. (2000) addressed a problem involving a very short time horizon. In that case, the heuristic that each product be shipped only once along the entire scheduling horizon is operationally convenient. For that reason, constraint 9 limits the number of times each product is loaded to pipelines along the scheduling horizon.

$$
\sum_{t \in T} Z_{j, p, t}^{S} \leq 1 \forall j \in J, p \in P
$$

Once a pumping operation is started it must also be finished within the time horizon as given by Equation 10 .

$$
\sum_{t \in T}\left(Z_{j, p, t}^{S}-Z_{j, p, t}^{E}\right)=0 \forall j \in J, p \in P
$$

Moreover, the time periods in which pumping has started and finished are identified by equations 11 and 12, respectively. It should be noted that, because of constraints 9 and 10 , only one of the $\mathrm{ZSj}, \mathrm{p}, \mathrm{t}$ in the summation on the right hand side of equation 11 will be nonzero. Likewise, only one of the ZEj,p,t in the summation on the right hand side of equation 12 will be nonzero. The inequality 13 ensures that the start of pumping will be no later than its end.

$$
\begin{gathered}
T_{j, p}^{S}=\sum_{t \in T} t \cdot Z_{j, p, t}^{S} \forall j \in J, p \in P \\
T_{j, p}^{E}=\sum_{t \in T} t \cdot Z_{j, p, t}^{E} \forall j \in J, p \in P \\
T_{j, p}^{S} \leq T_{j, p}^{E} \forall j \in J, p \in P
\end{gathered}
$$

Equation 14 sets the time interval in which product $p$ is being pumped. $\mathrm{Zj}, \mathrm{p}, \mathrm{t}=1$ means that pumping of product $\mathrm{p}$ is active in time period $t$ on pipeline $j$. Equation 14 sets the time-period interval in which product $\mathrm{p}$ is being blended and shipped. Equation (10) together with equation (14) prevents the situation where a product would start shipment and, before its completion, another product would start being shipped to the same pipeline.

$$
Z_{j, p, t}=\sum_{t^{\prime} \leq t} Z_{j, p, t^{\prime}}^{S}-\sum_{t^{\prime}<t} Z_{j, p, t^{\prime}}^{E} \forall j \in J, p \in P, t \in T
$$

If a tank is loading to a pipeline, then one of the products $\mathrm{p}$ is being pumped, as stated by constraint 15 . In addition, for a given pipeline and a given time period at most one of the products is being blended and loaded to the pipeline, constraint 16. 


$$
\begin{gathered}
Y_{i, j, t} \leq \sum_{p \in P} Z_{j, p, t} \forall i \in I, j \in J, t \in T \\
\sum_{p \in P} Z_{j, p, t} \leq 1 \forall j \in J, t \in T
\end{gathered}
$$

\section{d) Flowrate constraints}

Constraints 17, 18 and 19 impose limits to flowrate between column and tanks, between tanks and pipelines and through pipelines, respectively.

$$
\begin{gathered}
F_{i}^{\min } X_{i, t} \leq F_{i, t} \leq F_{i}^{\max } X_{i, t} \forall i \in I, t \in T \\
F_{i, j}^{\min } Y_{i, j, t} \leq F_{i, j, t} \leq F_{i, j}^{\max } Y_{i, j, t} \forall i \in I, j \in J, t \in T \\
F_{j}^{\min } Z_{j, p, t} \leq F_{j, p, t} \leq F_{j}^{\max } Z_{j, p, t} \forall i \in I, p \in P, t \in T
\end{gathered}
$$

\section{e) Transition constraints}

Transitions are known as the interfaces created between parcels of different products pumped consecutively through the same pipeline. Constraints 20 and 21 are complementary to each other and are used to identify potential transitions, given that each product is allowed to be pumped only once throughout the entire scheduling horizon.

$$
\begin{gathered}
P_{j, p, n} \geq \frac{T_{j, n}^{S}-T_{j, p}^{S}}{T} \forall j \in J, \quad p, n \in P, \quad p \neq n \\
-T\left(1-P_{j, p, n}\right) \leq T_{j, n}^{S}-T_{j, p}^{S} \quad \forall j \in J, \quad p, n \in P, \quad p \neq n
\end{gathered}
$$

A sample schedule is used to illustrate the application of the constraints 20 and 21 . Three products, $\mathbf{p 1}, \mathbf{p 2}$ and p3 are pumped through pipeline $\mathrm{j}$ along a time horizon composed of 8 discrete time-periods as given by Figure 2 . Pumping of product $\mathbf{p} \mathbf{1}$ starts at time-period 1 and finishes at time-period 3, pumping of product $\mathbf{p 2}$ starts at time period 4 and finishes at time-period 6 , whereas pumping of product $\mathbf{p} 3$ starts at time-period 7 and finishes at timeperiod 8. Therefore, $T_{j, p 1}^{S}=1, T_{j, p 1}^{E}=3, T_{j, p 2}^{S}=4$, $T_{j, p 2}^{E}=6, T_{j, p 3}^{S}=7$ and $T_{j, p 3}^{E}=8$. It should be noted that transitions are established between products $\mathbf{p} 1$ and $\mathbf{p} 2$ and between $\mathbf{p} 2$ and $\mathbf{p 3}$. However, there is no transition between $\mathbf{p} 1$ and $\mathbf{p 3}, \mathbf{p 2}$ and $\mathbf{p 1}, \mathbf{p 3}$ and $\mathbf{p 1}$ and $\mathbf{p 3}$ and $\mathbf{p 2}$.

\begin{tabular}{|c|c|c|c|c|c|c|c|}
\hline 1 & 2 & 3 & 4 & 5 & 6 & 7 & 8 \\
\hline $\mathbf{p 1}$ & $\mathbf{p 1}$ & $\mathbf{p 1}$ & $\mathbf{p 2}$ & $\mathbf{p 2}$ & $\mathbf{p 2}$ & $\mathbf{p 3}$ & $\mathbf{p 3}$ \\
\hline
\end{tabular}

Figure 2. Schedule involving pumping of three products (p1, p2 and $\mathbf{p 3}$ ) through a pipeline along a time horizon comprised of eight discrete time periods. 
Table 1. onstraints 20 and 21 reflecting the sample schedule of Figure 2.

\begin{tabular}{cccc}
\hline & Constraint 20 & \multicolumn{1}{c}{ Constraint 21} & $P_{j, p, n}$ \\
\hline $\mathbf{p 1}, \mathbf{p 2}$ & $P_{j, \mathbf{p} 1, \mathbf{p} 2} \geq(4-1) / 8=3 / 8$ & $-8\left(1-P_{j, \mathbf{p} 1, \mathbf{p} 2}\right) \leq(4-1)=3$ & 1 \\
$\mathbf{p 1}, \mathbf{p 3}$ & $P_{j, \mathbf{p} 1, \mathbf{p} 3} \geq(7-1) / 8=6 / 8$ & $-8\left(1-P_{j, \mathbf{p} 1, \mathbf{p} 3}\right) \leq(7-1)=6$ & 1 \\
$\mathbf{p 2}, \mathbf{p 1}$ & $P_{j, \mathbf{p} 2, \mathbf{p} 1} \geq(1-4) / 8=-3 / 8$ & $-8\left(1-P_{j, \mathbf{p} 2, \mathbf{p} 1}\right) \leq(1-4)=-3$ & 0 \\
$\mathbf{p 2}, \mathbf{p 3}$ & $P_{j, \mathbf{p} 2, \mathbf{p} 3} \geq(7-4) / 8=3 / 8$ & $-8\left(1-P_{j, \mathbf{p} 2, \mathbf{p} 3}\right) \leq(7-4)=3$ & 1 \\
$\mathbf{p 3}, \mathbf{p 1}$ & $P_{j, \mathbf{p} 3, \mathbf{p} 1} \geq(1-7) / 8=-6 / 8$ & $-8\left(1-P_{j, \mathbf{p} 3, \mathbf{p} 1}\right) \leq(1-7)=-6$ & 0 \\
$\mathbf{p 3}, \mathbf{p 2}$ & $P_{j, \mathbf{p} 3, \mathbf{p} 2} \geq(4-7) / 8=-3 / 8$ & $-8\left(1-P_{j, \mathbf{p} 3, \mathbf{p} 2}\right) \leq(4-7)=-3$ & 0
\end{tabular}

Constraints 20 and 21 are created for all combinations of $\mathbf{p} \mathbf{1}, \mathbf{p} \mathbf{2}$ and $\mathbf{p} \mathbf{3}$ as presented in Table 1. Note that the last column in the Table refers to the resulting values assumed by the variable $P_{j, p, n}$ when constraints 20 and 21 are simultaneously satisfied. The purpose of those constraints is to indicate potential transitions. For this reason, besides indicating the actual transitions between products $\mathbf{p} 1$ and p2 and between products $\mathbf{p 2}$ and $\mathbf{p 3}$, transition between products p1and $\mathbf{p 3}$ is also indicated as a potential one. Consequently, additional constraints must be introduced in order to screen out transitions that are not actual. However, before presenting the additional constraints, it must be emphasized that there is no transition when product $n$ equals product $p$, as postulated by Equation 22 .

$$
P_{j, p, n}=0 \quad \forall j \in J, p, n \in P, p=n
$$

Since each product can be handled only once along the entire time horizon and knowing that products for which there is no demand will not be shipped through pipelines (equation 6), it is possible to predict the total number of transitions at each pipeline. The total number of transitions will be equal to the total number of products for which there is demand diminished by 1 or null if demand is incurred for only one product, Equation 23. Note that Equation 23 is not part of the model but just the way parameter NTj is calculated and used as an input parameter to the model.

$$
N T_{j}=\max \left(\sum_{p \in P} Z D_{j, p}-1,0\right) \forall j \in J
$$

where, $Z D_{j, p}$ indicates if there is demand incidence for product $\mathrm{p}$ at pipeline $\mathrm{j}$. The input parameter NTj is used to define the actual total number of transitions allowed to be identified by the model, as given by Equation 24 .

$$
\sum_{p \in P} \sum_{n \in P} W_{j, p, n}=N T_{j} \forall j \in J
$$

Variable Wj,p,n assumes 1 if the actual transition is identified and 0 otherwise and it is upper bounded by $\mathrm{Pj}, \mathrm{p}, \mathrm{n}$ determined by constraints 20 and 21 . If there is no potential feasibility for the occurrence of a transition between products $\mathrm{p}$ and $\mathrm{n}(\mathrm{Pj}, \mathrm{p}, \mathrm{n}=0)$, then the actual transition will not be established between products $\mathrm{p}$ and $\mathrm{n}(\mathrm{Wj}, \mathrm{p}, \mathrm{n}=0)$, as guaranteed by constraint 25 . 


$$
W_{j, p, n} \leq P_{j, p, t} \forall j \in J, \quad p, n \in P
$$

As discussed before, constraints 20 and 21 usually indicate a number of potential transitions that is greater than or equal to the total number of the actual transitions.
Therefore, constraints 26 and 27 are added to screen out invalid transitions and to allow transitions between products $\mathrm{p}$ and $\mathrm{n}$ (or $\mathrm{n}$ and $\mathrm{p}$ ) to occur only once.

$$
\begin{aligned}
& \sum_{n \in P} W_{j, p, n} \leq Z D_{j, p} \quad \forall j \in J, \quad p \in P \\
& \sum_{p \in P} W_{j, p, n} \leq Z D_{j, n} \quad \forall j \in J, \quad n \in P
\end{aligned}
$$

Constraints 26 and 27 only allow transitions involving product $\mathrm{p}$ or $\mathrm{n}$ if there is demand incidence for them. In the model proposed by Pinto et al. (2000), the right hand side of constraints 26 and 27 was set to 1 instead of ZDj,p/ $\mathrm{ZDj}, \mathrm{n}$. That modification was necessary because, if there was no demand for a product, a value 1 on the right hand side of constraints 26 and 27, instead of $\mathrm{ZDj}, \mathrm{p} / \mathrm{ZDj}, \mathrm{n}$, could produce an erroneous transition identification, as we indeed found out.

In order to demonstrate the idea on how the set of constraints 26 and 27 work, we take again the example of Figure 2 and the potential candidates indicated in Table 1: $[(\mathbf{p} 1, \mathbf{p 2}),(\mathbf{p} 1, \mathbf{p 3}),(\mathbf{p} 2, \mathbf{p} 3)]$. The total number of transitions calculated by equation 23 is 2 , which is used in constraint 24 . Now, writing constraints 26 and 27 explicitly for all combinations of $p$ and $n$ we get Equations 26a-c and 27a-c shown in Table 2 as the individual constraints generated from constraint 26 and 27 defined over their domain, respectively.

Table2. Constraints 26 and 27 reflecting the sample schedule of Figure 2.

\begin{tabular}{ccc}
\hline $\mathbf{p} 1$ & Constraint 26 & Constraint \\
\hline $\mathbf{p} 2$ & $W_{j, \mathbf{p} 1, \mathbf{p} 1}+W_{j, \mathbf{p} 1, \mathbf{p} 2}+W_{j, \mathbf{p} 1, \mathbf{p} 3} \leq 1$ \\
$\mathbf{p} 3$ & $W_{j, \mathbf{p} 2, \mathbf{p} 1}+W_{j, \mathbf{p} 2, \mathbf{p} 2}+W_{j, \mathbf{p} 2, \mathbf{p} 3} \leq 1$ \\
& $W_{j, \mathbf{p} 3, \mathbf{p} 1}+W_{j, \mathbf{p} 3, \mathbf{p} 2}+W_{j, \mathbf{p} 3, \mathbf{p} 3} \leq 1$ \\
$\mathbf{p} 1$ & Constraint 27 \\
& $W_{j, \mathbf{p} 1, \mathbf{p} 1}+W_{j, \mathbf{p} 2, \mathbf{p} 1}+W_{j, \mathbf{p} 3, \mathbf{p} 1} \leq 1$ \\
$W_{j, \mathbf{p} 1, \mathbf{p} 2}+W_{j, \mathbf{p} 2, \mathbf{p} 2}+W_{j, \mathbf{p} 3, \mathbf{p} 2} \leq 1$ \\
$W_{j, \mathbf{p} 1, \mathbf{p} 3}+W_{j, \mathbf{p} 2, \mathbf{p} 3}+W_{j, \mathbf{p} 3, \mathbf{p} 3} \leq 1$
\end{tabular}

From 26a, either transition $[(\mathbf{p} 1, \mathbf{p} 2),(\mathbf{p} 1, \mathbf{p} 3)]$ is feasible. From 26b only transition (p2,p3) is possible, whereas $27 \mathrm{~b}$ allows only $(\mathbf{p} 1, \mathbf{p} 2)$ and by $27 \mathrm{c}$ either of the transitions $[(\mathbf{p} 1, \mathbf{p 3}),(\mathbf{p} 2, \mathbf{p 3})]$ is allowed. Therefore, the only solution that satisfies simultaneously 20-22 and 24-27 is the pair $[(\mathbf{p 1}, \mathbf{p 2}),(\mathbf{p} 2, \mathbf{p 3})]$, having in mind that the total number of transitions that must be identified is 2 .

A quick analysis of the just presented model lead us to conclude that this model, as is, cannot be readily applied to represent real-world problems encompassing a few days due to the fact that each product must be pumped at most once along the entire time horizon, which is reasonable for very short time horizons. If one aims at addressing longer time horizons, the whole portion of the model that represents the transition identification logic must be modified. In the present work, we aim at investigating alternative approaches for dealing with the pipeline schedule that will enable addressing problems with longerterm schedule horizons. 


\section{STUDIED APPROACHES}

Transitions arise naturally in scheduling problems and have been modeled and discussed in a number of PSE papers (Karamarkar and Schrage, 1985; Sahinidis and Grossmann, 1991; Kondili et al., 1993; Lee at al., 1996; Wolsey, 1997; Mendez et al., 2006; Kelly and Zyngier, 2007; Liu et al, 2010; Harjunkoski et al., 2014). Two contributions are worth bringing up in more details; Kondili et al. (1993), using the discrete time representation, introduced a mechanism for identifying transitions that relies on the evaluation of any two time-periods, as opposed to the traditional form in which only consecutive time-periods are evaluated (Lee at al., 1996). Kelly and Zienger (2007) proposed an alternative approach that uses auxiliary noninteger variables capable of producing tighter relaxation problems and thus resulting in a much more efficient approach than that of Kondili and coworkers.

In this section, two formulations are derived from the base model, which result from replacing the set of constraints that are used for identifying transitions by the two most common forms found in the literature, which consider evaluation of any two time-periods and evaluation of consecutive time-periods. The resulting formulations encompass very short time horizons in the same fashion as the base model. In that case, the adopted heuristic which dictates that products are handled only once at each pipeline is kept in the formulations without any hurdle. A new approach is then introduced that takes into consideration characteristics of the two classic forms of identifying transitions. Results obtained with the three formulations are compared and discussed in the results section. Next, attention is turned in the direction of problems addressing longer time horizons. In this case, improvements for the introduced approach are proposed that rely on use of two time scales, in which one can be derived as the aggregation of the other. The following sections are organized so that the discussion is concentrated in two different fronts; formulations for short-term time horizons and formulation for long-term time horizons. The complete set of equations that compose each optimization problem presented in the following sections are summarized in Appendix A1-A4. In all problems that follow, the objective function is given by Equation 28.

$$
\min \sum_{i \in I} \sum_{j \in J} \sum_{t \in T}\left(C r m_{i}+C p_{i}\right) F_{i, j, t}+\sum_{i \in I} \sum_{t \in T} C i_{i} V_{i, t}+\sum_{j \in J} \sum_{p \in P} \sum_{n \in P, p \neq n e \in E} \sum_{p, n} W_{j, p, n, e}
$$

\section{Short-Term Time Horizon}

Model 1

In the first approach, the variable $P_{j, p, n}$ together with constraints 20-22 and 25-27 are dropped from the base model, which are replaced by constraint 29 . This constraint has been extensively used in optimization formulations for identifying transitions between tasks occurring in consecutive time-periods (see for example Lee at al., 1996).

$$
W_{j, p, n} \geq Z_{j, p, t}+Z_{j, n, t+1}-1 \forall j \in J, \quad p, n \in P, t \in T, p \neq n
$$

The reader should notice that 29 causes an expressive increase in the number of constrains, since an equation is created for every $(p, n)$ combination between two time periods, $\mathrm{t}$ and $\mathrm{t}+1$, with $p \neq n$. On the other hand, there is also a reduction in the number of constraints and variables by dropping constraints $20-22$ and 25-27 and variables $\mathrm{Pj}, \mathrm{p}, \mathrm{n}$.

There is an evident flaw in this approach in that constraint 29 is only capable of identifying transitions in cases where different tasks are allocated to adjacent time-periods. Therefore, it is not of practical use unless allocation is enforced for every time-period or if idle time periods are not intermediary ones. In spite of that, this approach is kept in our studies for sake of comparison with other approaches.

Model 2
By the discussion of the last section, it can be concluded that constraint 29 cannot be used as a mechanism to identify transitions for the pipeline-scheduling problem, given that there might well be time-periods for which there will be no allocation. The beauty of constraint 29 , though, is that it is simple and it can be modified to tackle situations where tasks are allocated to time-periods that are far apart and no tasks are scheduled in between. As already cited, Kondili et al. (1993) have proposed a different form of 29, constraint 30 . The first modification one can see is related to the time-periods involved in the evaluation. Instead of considering only adjacent time periods $t$ and $t+1$, any two time periods $t$ and $t$ ' are taking into account, requiring only that $t^{\prime}>t$. Another modification is also made to guarantee that the new constraint is always satisfied. The third term on the right hand side of 30 is null in case no tasks are 
scheduled in between the time-periods under evaluation and thus $W_{j, p, n}=1$ will be enforced given that $p$ is allocated to $t$ and $n$ is allocated to $t^{\prime}$. If, on the other hand, at least one task is scheduled in between $t$ and $t^{\prime}$, the third term on the right hand side of 30 will result in an integer number, leading to the relaxation of the constraint, regardless of the allocations in $t$ and $t^{\prime}$.

$$
W_{j, p, n} \geq Z_{j, p, t}+Z_{j, n, t^{\prime}}-\sum_{p^{\prime} \in P} \sum_{t^{\prime \prime}=t+1}^{t^{\prime}-1} Z_{j, p^{\prime}, t^{\prime \prime}}-1 \forall j \in J, p, n \in P, t \in T, p \neq n
$$

An apparent drawback of constraint 30 in comparison to 29 is the total number of constraints necessary for creating all combinations of $\left(t, t^{\prime}\right)$ and $(p, n)$. Note that for each $t, t$ ' will be varied from $t+1$ to the last time-period of the scheduling horizon and hence creating a huge number of constraints.

Figure 3 illustrates the use of constraint 30 in one such example where the pumping operation for product $\mathbf{p} \mathbf{1}$ is scheduled to start in the first time-period and to end in the fourth time period. Pipeline operation is then temporarily interrupted for the next two time-periods. Pumping of product $\mathbf{p 2}$ is initiated in the seventh time-period and continues until the end of the schedule horizon. In Table 3 , constraint 30 is illustrated for $t=4$, with $t$ ' varying from 5 to $8, p=\mathbf{p 1}$ and $n=\mathbf{p 2}$. It should be noted that, for $t$ ' $=t+1$ (adjacent time periods), 30 assumes the form of constraint 29. It is also demonstrated that 30 is able to handle pumping interruption and still identify transitions correctly. It must be borne in mind that constraint 30 works in synchronization with the objective function in that $W_{j, p, n}$ $=1$ incurs transition costs. Therefore, $W_{j, p, n}$ will be pushed down to zero by the objective function in case constraint 30 does not impose that $W_{j, p, n}=0$.

\begin{tabular}{|c|c|c|c|c|c|c|c|c|c|}
\hline 1 & 2 & 3 & 4 & 5 & 6 & 7 & 8 & 9 & 10 \\
\hline $\mathbf{p} 1$ & $\mathbf{p} 1$ & $\mathbf{p} 1$ & $\mathbf{p} 1$ & & & $\mathbf{p 2}$ & $\mathbf{p 2}$ & $\mathbf{p 2}$ & $\mathbf{p 2}$ \\
\hline
\end{tabular}

Figure 3. Schedule involving pumping of two products ( $\mathrm{p} 1$ and $\mathrm{p} 2$ ) through a pipeline along a time horizon comprised of ten discrete time periods.

Table 3. Constraints 30 reflecting the sample schedule of Figure 3.

\begin{tabular}{cc}
\hline Constraint 30 & $W_{j, \mathbf{p} 1, \mathbf{p} 2}$ Constraint \\
\hline$W_{j, \mathbf{p} 1, \mathbf{p} 2} \geq Z_{j, \mathbf{p} 1,4}+Z_{j, \mathbf{p} 2,5}-1$ & 0 \\
$W_{j, \mathbf{p} 1, \mathbf{p} 2} \geq Z_{j, \mathbf{p} 1,4}+Z_{j, \mathbf{p} 2,6}-\left(Z_{j, \mathbf{p} 1,5}\right)-\left(Z_{j, \mathbf{p} 2,5}\right)-1$ & 0 \\
$W_{j, \mathbf{p} 1, \mathbf{p} 2} \geq Z_{j, \mathbf{p} 1,4}+Z_{j, \mathbf{p} 2,7}-\left(Z_{j, \mathbf{p} 1,5}+Z_{j, \mathbf{p} 1,6}\right)-\left(Z_{j, \mathbf{p} 2,5}+Z_{j, \mathbf{p} 2,6}\right)-1$ & 1 \\
$W_{j, \mathbf{p} 1, \mathbf{p} 2} \geq Z_{j, \mathbf{p} 1,4}+Z_{j, \mathbf{p} 2,8}-\left(Z_{j, \mathbf{p} 1,5}+Z_{j, \mathbf{p} 1,6}+Z_{j, \mathbf{p} 1,7}\right)-\left(Z_{j, \mathbf{p} 2,5}+Z_{j, \mathbf{p} 2,6}+Z_{j, \mathbf{p} 2,7}\right)-1$ & 0 \\
\hline
\end{tabular}

Table 4. Constraints 32 and 33 reflecting the sample schedule of Figure 3.

\begin{tabular}{cc}
\hline Constraint (32) & Constraint (32) excluded null terms \\
\hline$\sum_{n \in P} Z_{j, n, 5}+S_{j, \mathbf{p} 1,5} \geq Z_{j, \mathbf{p} 1,4}+S_{j, \mathbf{p} 1,4}$ & $S_{j, \mathbf{p} 1,5} \geq Z_{j, \mathbf{p} 1,4}$ \\
$\sum_{n \in P} Z_{j, n, 6}+S_{j, \mathbf{p} 1,6} \geq Z_{j, \mathbf{p} 1,5}+S_{j, \mathbf{p} 1,5}$ & $S_{j, \mathbf{p} 1,6} \geq S_{j, \mathbf{p} 1,5}$ \\
$\sum_{n \in P} Z_{j, n, 7}+S_{j, \mathbf{p} 1,7} \geq Z_{j, \mathbf{p} 1,6}+S_{j, \mathbf{p} 1,6}$ & $\sum_{n \in P} Z_{j, n, 7} \geq S_{j, \mathbf{p} 1,6}$
\end{tabular}


Table 4. Cont.

\begin{tabular}{ccc}
\hline Constraint (33) & Constraint (33) excluded null terms & $W_{j, \mathbf{p 1} \mathbf{p} 2}$ \\
\hline$W_{j, \mathbf{p} 1, \mathbf{p} 2} \geq Z_{j, \mathbf{p} 1,4}+S_{j, \mathbf{p} 1,4}+Z_{j, \mathbf{p} 2,5}-1$ & $W_{j, \mathbf{p} 1, \mathbf{p} 2} \geq Z_{j, \mathbf{p} 1,4}-1$ & 0 \\
$W_{j, \mathbf{p} 1, \mathbf{p} 2} \geq Z_{j, \mathbf{p} 1,5}+S_{j, \mathbf{p} 1,5}+Z_{j, \mathbf{p} 2,6}-1$ & $W_{j, \mathbf{p} 1, \mathbf{p} 2} \geq S_{j, \mathbf{p} 1,5}-1$ & 0 \\
$W_{j, \mathbf{p} 1, \mathbf{p} 2} \geq Z_{j, \mathbf{p} 1,6}+S_{j, \mathbf{p} 1,6}+Z_{j, \mathbf{p} 2,7}-1$ & $W_{j, \mathbf{p} 1, \mathbf{p} 2} \geq S_{j, \mathbf{p} 1,6}+Z_{j, \mathbf{p} 2,7}-1$ & 1 \\
\hline
\end{tabular}

\section{Model 3}

If on the one hand constraint 29 is simple and easy to apply, on the other hand it is not able to identify transitions if allocation of intermediary time-periods is vacant. Constraint 30 is able to circumvent the downside of constraint 29 but at the expense of an increase in the number of constraints, which affect computational performance. We propose a third approach that is conceptually similar to the first approach in that only consecutive time-periods are evaluated but some kind of mechanism needs to be added to address the case of idle time-periods. The solution found was to keep track of the history on the last product loaded to the pipeline, in case the pipeline operation is interrupted temporarily. That information is then used to correctly identify transitions under any circumstances. This idea of using memory variables has been used by other researchers in the past (Kelly and Zyngier, 2007), but our formulation is completely different.

In order to be able to track history, a new variable is introduced, $S_{j, p, t}$, which assumes 1 if operation of pipeline $j$ is interrupted at time period $t$ and the last product loaded to the pipeline was $p$. In the context of the pipeline operation schedule, at any point in time a pipeline might be either pumping a single product or idle, as stated in Equation 31. The second summation on the left hand side of the equation will be nonzero if the pipeline operation was interrupted in time-period $t$ and one of the products $p$ was the last product injected into the pipeline sometime in the past.

$$
\sum_{p \in P} Z_{j, p, t}+\sum_{p \in P} S_{j, p, t}=1 \forall j \in J, t \in T
$$

Constraint 32 identifies the exact time-period in which the last injection was done. If product $p$ is shipped in timeperiod $\mathrm{t}, \mathrm{Zj}, \mathrm{p}, \mathrm{t}=1$ and $\mathrm{Sj}, \mathrm{p}, \mathrm{t}=0$ by 31 . If operation is interrupted in the following time period $\sum_{n \in P} Z_{j, n, t+1}=0$ constraint 32, which then enforces $\mathrm{Sj}, \mathrm{p}, \mathrm{t}+1=1$, meaning that the information on $\mathrm{p}$ as the last loaded product will be carried over. If the pipeline remains static for more than one time-period $\mathrm{Sj}, \mathrm{p}, \mathrm{t}$ will repeatedly pass the information on to $\mathrm{Sj}, \mathrm{p}, \mathrm{t}+1$ until pipeline operation is recovered or the end of the scheduling horizon is reached. Transition is then easily identified by evaluating adjacent time-periods according to constraint 33 . Note that the only difference between constraints 29 and 33 is the introduction of $\mathrm{Sj}, \mathrm{p}, \mathrm{t}$ on the right hand side. However, this constraint works in coordination with constraints 31 and 32 .

$$
\begin{gathered}
\sum_{n \in P} Z_{j, n, t+1}+S_{j, p, t+1} \geq Z_{j, p, t}+S_{j, p, t} \forall j \in J, p \in P, t \in T-\{\mathrm{T}\} \\
W_{j, p, n} \geq Z_{j, p, t}+S_{j, p, t}+Z_{j, n, t+1}-1 \forall j \in J, \quad p, n \in P, t \in T-\{\mathrm{T}\}, p \neq n
\end{gathered}
$$

Constraints 31 and 32 are illustrated with the example of Figure 3 (Table 4) along time-periods 4, 5 and 6 comprising the time interval in which the pipeline operation is interrupted. Constraint 32 is written only for product $\mathbf{p 1}$, whereas constraint 33 is written for the transition identification between products $\mathbf{p 1}$ and $\mathbf{p 2}$, which is effectively done even with the pipeline remaining without operation for two consecutive time-periods.

\section{Long-Term Time Horizon}

The base model was built based on three fundamental heuristics: 1) products are shipped only once at each pipeline along the entire scheduling horizon; 2) the total number of transitions is known beforehand through constraint 23 and used in constraint 24; and 3) only products with nonzero demand are accounted for in constraints 26 and 27, which 
are used to screen out non-existing transitions potentially identified by constraints 20 and 21 .

In principle, for long time horizons, the idea that products are shipped only once along the entire time horizon cannot be sustained since multiple shipments are unavoidable because demand is distributed along the time horizon and pipelines are capacitated. In other words, there may be multiple due dates for the same product and, most likely, it will not always be possible to lump parcels so as to fulfill multiple demand incidences because of pumping capacity. On the other hand, enforcing a maximum number of shipments for each product establishes the maximum number of events or shipments that may happen within a time interval, which sets an upper bound on the number of transitions. In order to take advantage of this fact, the idea was to create two levels of granularity for time, e.g., the scheduling horizon could be subdivided in days within which products could be allowed to be shipped only once, which in turn could be subdivided in hours to accommodate allocation of multiple time periods. Bottom line, with the two levels of aggregation, transitions would be managed on a higher level of detail and hence a few constraints would be required to create all combinations of product interfaces and product allocation would be managed on the lower level of aggregation.

As to the second heuristic in which the total number of transitions is known beforehand, under no conditions could it be sustained in longer scheduling horizons because shipment of products could be anticipated, including products for which there was no demand in previous days. As long as there was available free capacity, anticipation would be possible. Shipment of products with zero demand could be convenient in cases of pipelines that transport multiple products and require more flexibility in terms of product sequencing. In that case, a small volume of a product with no demand could be injected in between products that would otherwise not be allowed to be put in contact with each other, which is sometimes a common practice.

In the previous section three different approaches were considered, all of which involve replacement of constraints 20-22 and 25-27 by other forms of transition identification. As already stated and, as will be demonstrated by the results presented below, the first approach is not robust enough to address scenarios where interruptions are scheduled between shipments. The second approach produces models with dimensions that grow very quickly with the scheduling horizon. The third approach was then readjusted to incorporate the time aggregation scheme mentioned previously so that that approach can be extended to cases where longer time horizons are considered.

\section{Model 4}

In this approach, the fundamental premise that each product can be shipped only once at each pipeline is retained in the model, not for the entire scheduling horizon but for a predefined set of time-periods. The scheduling horizon is subdivided into two levels of granularity. In the lowest level, the scheduling horizon is split in timeperiods representing hour buckets. Time-periods are then aggregated in day buckets. There is a subset of time-periods

that belongs to each day ( $t \in T_{d}$ ), as illustrated in Figure 4. With this bi-level time representation, it is imposed that the same product cannot be shipped more than once within the same day. Note also, by the scheme presented in Figure 4 , that event points are created to either indicate points in time in which the start of new shipments is scheduled or the start of a new day. The number of event points within each day must equal the number of products and the total number of event points must be $|\mathbf{E}|=|\mathbf{P}|$.|D $\mid$. A subset of event points is allocated to each day in increasing

order $\left(e \in E_{d}\right)$ so that the event point subsets are mutually exclusive. With that framework, transitions are identified by comparing adjacent events $e$ and $e+1$, even if they belong to different days.

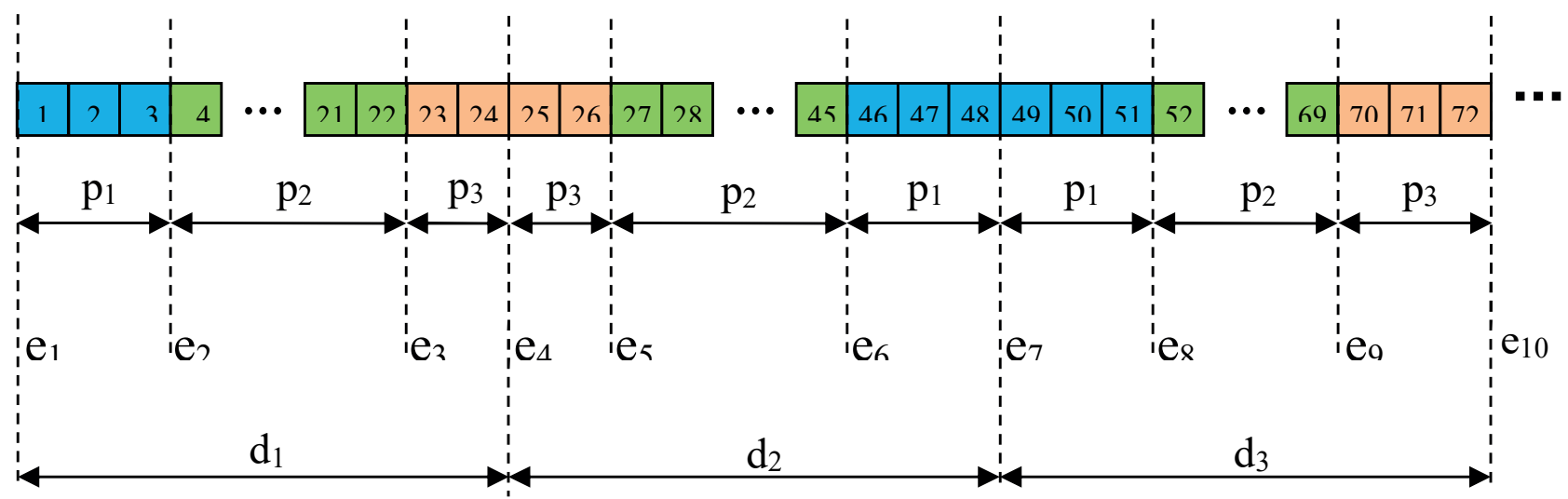

Figure 4. Event point - bilevel time representation. 
Most of the constraints indexed in time remain unchanged. The only constraints that are impacted by the new time representation are those representing operating rules used for ensuring that there will be single movements for each product within the same day. Therefore, constraints 34-38 are the equivalent of Equations 9-12 and 14 indexed only in time-periods, which represent hours. Constraint 13 is replaced by Equation 39 to ensure that the end of pumping will be equal to its start plus the pumping duration.

$$
\begin{gathered}
\sum_{t \in T_{d}} Z_{j, p, t, d}^{S} \leq 1 \forall j \in J, p \in P, d \in D \\
\sum_{t \in T_{d}}\left(Z_{j, p, t, d}^{S}-Z_{j, p, t, d}^{E}\right)=0 \forall j \in J, p \in P, d \in D \\
T_{j, p, d}^{S}=\sum_{t \in T_{d}} t \cdot Z_{j, p, t, d}^{S} \forall j \in J, p \in P, d \in D \\
T_{j, p, d}^{E}=\sum_{t \in T_{d}} t \cdot Z_{j, p, t, d}^{E} \forall j \in J, p \in P, d \in D \\
Z_{j, p, t}=\sum_{t^{\prime} \leq t} Z_{j, p, t^{\prime}, d}^{S}-\sum_{t^{\prime}<t} Z_{j, p, t^{\prime} d}^{E} \forall j \in J, p \in P, t \in T_{d}, d \in D \\
T_{j, p, d}^{E}=T_{j, p, d}^{S}+\left(\sum_{t \in T_{d}} Z_{j, p, t}-1\right), \quad \forall j \in J, p \in P, d \in D
\end{gathered}
$$

Equation 6 must be decomposed into two equations if demand is to be met exactly. Equation 40a guarantees that demand is satisfied at due dates, whereas $40 \mathrm{~b}$ enforces that the total amount of product $p$ shipped along the scheduling horizon must exactly meet demand. Note that products with no demand are allowed to be shipped since constraint $40 \mathrm{~b}$ is applied only for those products with non-zero demand. If the total volume transferred of each product were allowed to be greater than the total demand, $40 \mathrm{~b}$ would be modified with a corresponding greater than or equal sign.

$$
\begin{gathered}
\sum_{t^{\prime} \leq t} F_{j, p, t^{\prime}} \geq \sum_{t^{\prime} \leq t} D_{j, p, t^{\prime}} \forall j \in J, p \in P, t \in T, N T_{j}>1 \\
\sum_{t} F_{j, p, t}=\sum_{t} D_{j, p, t} \forall j \in J, p \in P, t \in T
\end{gathered}
$$

The start of a pumping operation must match an event point, which is done through Equation 41. The instant of time in which the event occurs must exactly match the start of the pumping operation, (constraints 42 and 43 ).

$$
\begin{array}{cl}
\sum_{e \in E_{d}} E_{j, p, e}=\sum_{t \in T_{d}} Z_{j, p, t, d}^{S} & \forall j \in J, p \in P, d \in D \\
T_{j, e} \geq T_{j, p, d}^{S}-H_{d}\left(1-E_{j, p, e}\right) & \forall j \in J, p \in P, e \in E_{d}, d \in D \\
T_{j, e} \leq T_{j, p, d}^{S}+H_{d}\left(1-E_{j, p, e}\right) & \forall j \in J, p \in P, e \in E_{d}, d \in D
\end{array}
$$

Event points must be monotonically non-decreasing, identification constraints, which is guaranteed by Equation since adjacent event points are used in transition 44. 


$$
T_{j, e} \geq T_{j, e-1} \quad \forall j \in J, e \in E
$$

There might be event points to which no pumping operation will be allocated. With the intent of avoiding the occurrence of symmetric solutions, constraint 45 is added. By introducing such a constraint, pumping operations will be always forced to be allocated to the first events of each day, which has a direct impact on computational performance.

$$
\sum_{p \in P} E_{j, p, e} \geq \sum_{p \in P} E_{j, p, e+1} \quad \forall j \in J, d \in D, e \in E_{d}^{L}
$$

Transitions are identified considering only consecutive event points, instead of consecutive time-periods, which drastically reduces model size in comparison to the case where time-periods are used. The set of constraints involved in transition identification is similar to that proposed in model 3. The only difference is that, instead of time-periods, event points are considered (constraints 46-48).

$$
\begin{gathered}
\sum_{p \in P} E_{j, p, e}+\sum_{p \in P} S_{j, p, e}=1 \quad \forall j \in J, e \in E \\
\sum_{n \in P} E_{j, n, e+1}+S_{j, p, e+1} \geq E_{j, p, e}+S_{j, p, e} \quad \forall j \in J, p \in P, e \in E-\{\mathrm{E}\} \\
W_{j, p, n, e} \geq E_{j, p, e}+S_{j, p, e}+E_{j, n, e+1}-1 \forall j \in J, p, n \in P, e \in E-\{\mathrm{E}\}, p \neq n
\end{gathered}
$$

The complete model is presented in Appendix A4.

\section{NUMERICAL RESULTS}

The problem presented in Pinto et al. (2000) was used, with minor changes, for illustrating the application of the first three approaches, which comprises a very shortterm time horizon comprised of 24 uniform time-periods of 1 hour. A derivation of that example was also used to illustrate the application of the extended approach, which comprises a time horizon of 4 days (96 hours). For the short-term schedule, the volume injected into the pipeline is allowed to be greater than demand, which is incurred only at the end of the time horizon, whereas demand is distributed along the time horizon for the extended one with incurrence at the end of each day. Demand data are given in Table 5. Three distillation columns produce intermediate products with different constant properties, which are stored in dedicated tanks. The input data related to distillation columns, rundown tanks and pumping capacities are given in Table 6 . The intermediate products are blended in-line to produce diesel with three different specs (Table 7) and dispatched through three pipelines. Full connectivity is assumed. Dependent transition costs are given in Table 8.

All formulations resulted in MILP problems which were coded using the GAMS 24.4 system and solved by
CPlex 12 on an Intel(R) Core(TM)i7, CPU3.5 GHz and 16.0GB RAM. The relative gap was set to $0.01 \%$ as one of the termination criteria for the approaches involving shortterm time horizons and $0.1 \%$ or $3,600 \mathrm{CPU}$ seconds for the approach encompassing longer time horizon.

\section{Short-Term Time Horizon}

The computational results for all approaches encompassing short-term time horizons are presented in Table 9, from which it can be noticed that the first and second approaches contain less binary variables than the base model since the variable $P_{j, p, n}$ was dropped in the new approaches. However, the introduction of $S_{j, p, t}$ variable in the third approach contributed to a slight increase in the number of binary variables.

Constraints 20-22 and 24-27 of the base model were replaced by different constraints depending on the approach. For model 1, there was a net increase of $11 \%$ in the number of constraints due to the use of constraint 29 as the mechanism for identifying transitions, whereas the net increase for model 3 was $20 \%$ due to the set of constraints 31-33. For model 2 the number of constraints was 2.6 times the number of constrains of the base model. The introduction of constraint 30 added $|\mathbf{T}|-t$ new 
A Novel Transition Identification Mechanism for the Diesel Blending and Distribution Scheduling Problem Using the Discrete Time Representation with Two Time-Scales Granularity

Table 5. Demand data $\left(\times 10^{-3} \mathrm{~m}^{3}\right)$

\begin{tabular}{|c|c|c|c|c|}
\hline & \multicolumn{4}{|c|}{ Time horizon (24 hours) } \\
\hline & $\mathrm{t}$ & Pipeline 1 & Pipeline 2 & Pipeline 3 \\
\hline D1 & 24 & 4.0 & 2.5 & 1.5 \\
\hline D2 & 24 & 3.0 & 3.5 & 2.0 \\
\hline \multirow[t]{5}{*}{ D3 } & 24 & 1.0 & 3.0 & 2.0 \\
\hline & \multicolumn{4}{|c|}{ Time horizon (96 hours) } \\
\hline & $\mathrm{t}$ & Pipeline 1 & Pipeline 2 & Pipeline 3 \\
\hline & 24 & 1.8 & 2.0 & 0.0 \\
\hline & 48 & 3.5 & 1.8 & 1.2 \\
\hline \multirow[t]{2}{*}{ D1 } & 72 & 0.0 & 3.4 & 1.3 \\
\hline & 96 & 0.0 & 0.0 & 1.2 \\
\hline \multirow{4}{*}{ D 2} & 24 & 2.0 & 2.5 & 2.0 \\
\hline & 48 & 0.0 & 3.2 & 1.6 \\
\hline & 72 & 3.4 & 0.0 & 2.5 \\
\hline & 96 & 2.6 & 2.8 & 2.2 \\
\hline \multirow{4}{*}{ D 3} & 24 & 1.5 & 2.7 & 1.5 \\
\hline & 48 & 3.0 & 0.0 & 2.3 \\
\hline & 72 & 3.2 & 3.1 & 1.9 \\
\hline & 96 & 2.4 & 3.2 & 0.0 \\
\hline
\end{tabular}

Table 6. Parameters related to the production of distillation columns, tank capacities and flowrate bounds.

\begin{tabular}{|c|c|c|c|c|c|c|}
\hline & Tank 1 & Tank 2 & Tank 3 & Tank 4 & Tank 5 & Tank 6 \\
\hline Sulfur (wt. \%) & 0.30 & 0.30 & 0.60 & 0.40 & 1.00 & 1.00 \\
\hline Cetane number & 42.0 & 42.0 & 40.3 & 39.0 & 40.0 & 40.0 \\
\hline$V_{i}^{\min } \times 10^{3}\left(\mathrm{~m}^{3}\right)$ & 2 & 2 & 2 & 2 & 2 & 2 \\
\hline$V_{i}^{\max } \times 10^{3}\left(\mathrm{~m}^{3}\right)$ & 30 & 30 & 30 & 30 & 30 & 30 \\
\hline$V_{i}^{0} \times 10^{3}\left(\mathrm{~m}^{3}\right)$ & 10 & 20 & 8 & 8 & 15 & 12 \\
\hline$F_{i}^{\min }\left(\mathrm{m}^{3} / \mathrm{h}\right)$ & 250 & 250 & 220 & 220 & 180 & 180 \\
\hline$F_{i}^{\max }\left(\mathrm{m}^{3} / \mathrm{h}\right)$ & 300 & 300 & 250 & 250 & 200 & 200 \\
\hline$F_{i j}^{\min }\left(\mathrm{m}^{3} / \mathrm{h}\right)$ & 30 & 30 & 40 & 40 & 40 & 40 \\
\hline$F_{i j}^{\max }\left(\mathrm{m}^{3} / \mathrm{h}\right)$ & 500 & 500 & 500 & 500 & 500 & 500 \\
\hline$C i_{i}\left(\$ / \mathrm{m}^{3}\right)$ & 0.10 & 0.10 & 0.12 & 0.12 & 0.11 & 0.11 \\
\hline$C p_{i}\left(\$ / \mathrm{m}^{3}\right)$ & 0.20 & 0.20 & 0.18 & 0.18 & 0.16 & 0.16 \\
\hline $\mathrm{Crm}_{i}\left(\$ / \mathrm{m}^{3}\right)$ & 0.60 & 0.60 & 0.40 & 0.40 & 0.05 & 0.05 \\
\hline
\end{tabular}

Table 7. Diesel specs.

\begin{tabular}{ccc}
\hline Diesel & Sulfur & Cetane \\
Grade & (wt. \%) & Number \\
\hline D1 & 0.3 & 42 \\
D2 & 0.5 & 40 \\
D3 & 1.0 & 40 \\
\hline
\end{tabular}

Table 8. Transition costs (\$).

\begin{tabular}{lccc}
\hline & D1 & D2 & D3 \\
\hline D1 & - & 110 & 100 \\
D2 & 130 & - & 120 \\
D3 & 190 & 190 & - \\
\hline
\end{tabular}


constraints for each $t$ causing the most significant increase in the number of constraints and the worst computational performance. Another important fact that needs to be brought up is about the quality of the relaxed problem produced by each formulation. The formulation proposed by Pinto et al. (2000) is able to produce good relaxation, whereas all other formulations produce poorly relaxed problems. This explains why the base model outperforms all other evaluated formulations in terms of required number of iterations, nodes and CPU time for solving the same problem.

Table 9. Computational statistics -Short-term time horizon

\begin{tabular}{|c|c|c|c|c|}
\hline & Base model & Model 1 & Model 2 & Model 3 \\
\hline Equations & 3,028 & 3,367 & 7,903 & 3,646 \\
\hline Binary variables & 1,278 & 1,242 & 1,242 & 1,458 \\
\hline Continuous variables & 2,233 & 2,197 & 2,197 & 2,413 \\
\hline Objective function (\$) & 880.18 & 421.29 & 880.20 & 880.18 \\
\hline Best bound & 880.09 & 421.26 & 880.11 & 880.09 \\
\hline $\mathrm{CPU}(\mathrm{s})$ & 10.86 & 13.84 & 163.29 & 34.23 \\
\hline Relative gap (\%) & 0.01 & 0.01 & 0.01 & 0.01 \\
\hline $\mathrm{B} \& \mathrm{~B}$ root solution & 879.23 & 189.33 & 176.16 & 189.22 \\
\hline Time for $1^{\text {st }}$ int. sol. (gap) & $0.91(92.85)$ & $1.23(73.96)$ & $2.23(81.39)$ & $1.12(81.40)$ \\
\hline Nodes & 3,630 & 6,315 & 22,599 & 115,750 \\
\hline Iterations & 63,955 & 130,825 & $1,105,549$ & 799,817 \\
\hline Material cost (\$) & 9.12 & 9.06 & 9.14 & 9.12 \\
\hline Pumping cost (\$) & 4.44 & 4.27 & 4.44 & 4.44 \\
\hline Inventory cost (\$) & 176.62 & 177.96 & 176.62 & 176.62 \\
\hline Transition cost $(\$)$ & 690.00 & 230.00 & 690.00 & 690.00 \\
\hline
\end{tabular}

In terms of the objective function, it can be seen that models 2 and 3 produced the same result as the one obtained for the base model, which can be verified by the cost components. Model 2 presented little difference in terms of cost components. Transition cost represents, by far, the biggest chunk of the overall objective function. Models 2 and 3 produced the same total transition cost as that of the base model. However, the disparate transition cost seen in Table 9 for model 1 is not an actual value since not all transitions were recognized. Some of the transitions were not accounted for since constraint 29 only works when nonempty adjacent time-periods are evaluated. Consequently, optimization wisely allocated an empty time period between each transition so that no cost would be incurred. This fact leads us to conclude that constraint 29 can indeed only be effective in cases where full time operation is enforced or non-allocated time-periods are all left to the beginning or the end of the scheduling horizon. The computational time of models 1 and 3 were in the same range as that of the base model. Model 2, on the other hand, was the less efficient approach due to the huge increase in model size and it is evident that this approach is not a feasible option for problems involving too many timeperiods.

Figure 5 provides the Gantt chart for all approaches. Products are identified by different colors (D1 - blue, D2 - green, D3 - orange) and the total transferred volumes are shown on top of the bars. It should be noted that, with exception of model 1, all approaches produced the same product sequence and total transferred volumes, which were greater than the minimal demand. From the refinery perspective, this strategy induces inventory reduction, which is the second most important cost component in the objective function. Moreover, it avoids interruptions in pipeline operation, which would otherwise promote the undesired mixing of the different product parcels. Although model 1 was able to meet demand and produce almost the same product sequence, transitions were only identified when pipelines were continuously operated (pipeline 2), which cannot always be guaranteed.

Figures 6-9 present flowrate profiles. These pictures show that pipelines are scheduled to operate at maximum capacity for most of the time, which is in accordance with the idea of pursuing minimal inventory at the refinery storage tanks. Once again, it should be noted that model 2, model 3 and the base model produced exactly the same solution as to the pipeline operation. The differences in solution rely on the blending operation. Figures 10-13 comprise inventory profiles in storage tanks (a) and flowrates established from tanks and pipelines (b), respectively. Here, pipelines to which tanks are connected in each time-period are illustrated in different colors (J1 - blue, J2 - green, J3 yellow). Solid colors mean even tanks whereas pattern filled bars mean odd tanks, having in mind that there are a couple of tanks available for each intermediate product. It is important to highlight that it makes no difference which 
of the intermediate tanks is used in the blending operation since their content is essentially the same. Having said that, it should be noted for the same pipeline schedule there are many possible symmetric solutions. Therefore, it can be stated that the solutions produced by model 2, model 3 and the base model are alternative solutions.
Given that tanks are not allowed to load and unload simultaneously and operation of the distillation units are continuous throughout the entire schedule horizon, the tank receiving intermediate material from the unit cannot be used for blending, which is indeed satisfied and observed by the positive or negative slopes of Figures 10-13 (a).

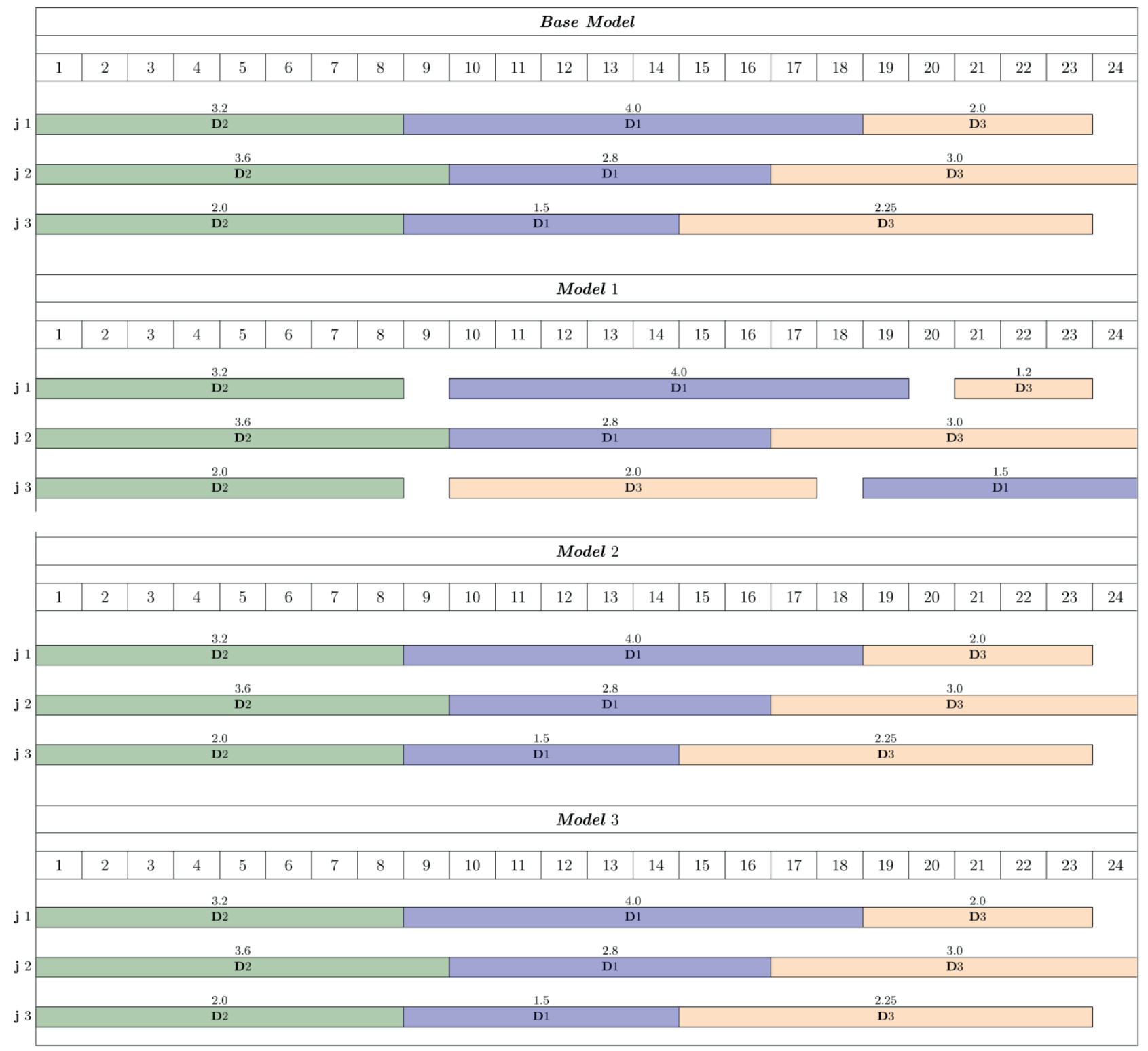

Figure 5. Gantt Charts obtained with the Base Model and Models 1-3 for the short-term time horizon (D1 - blue, D2 - green, D3 - orange). 

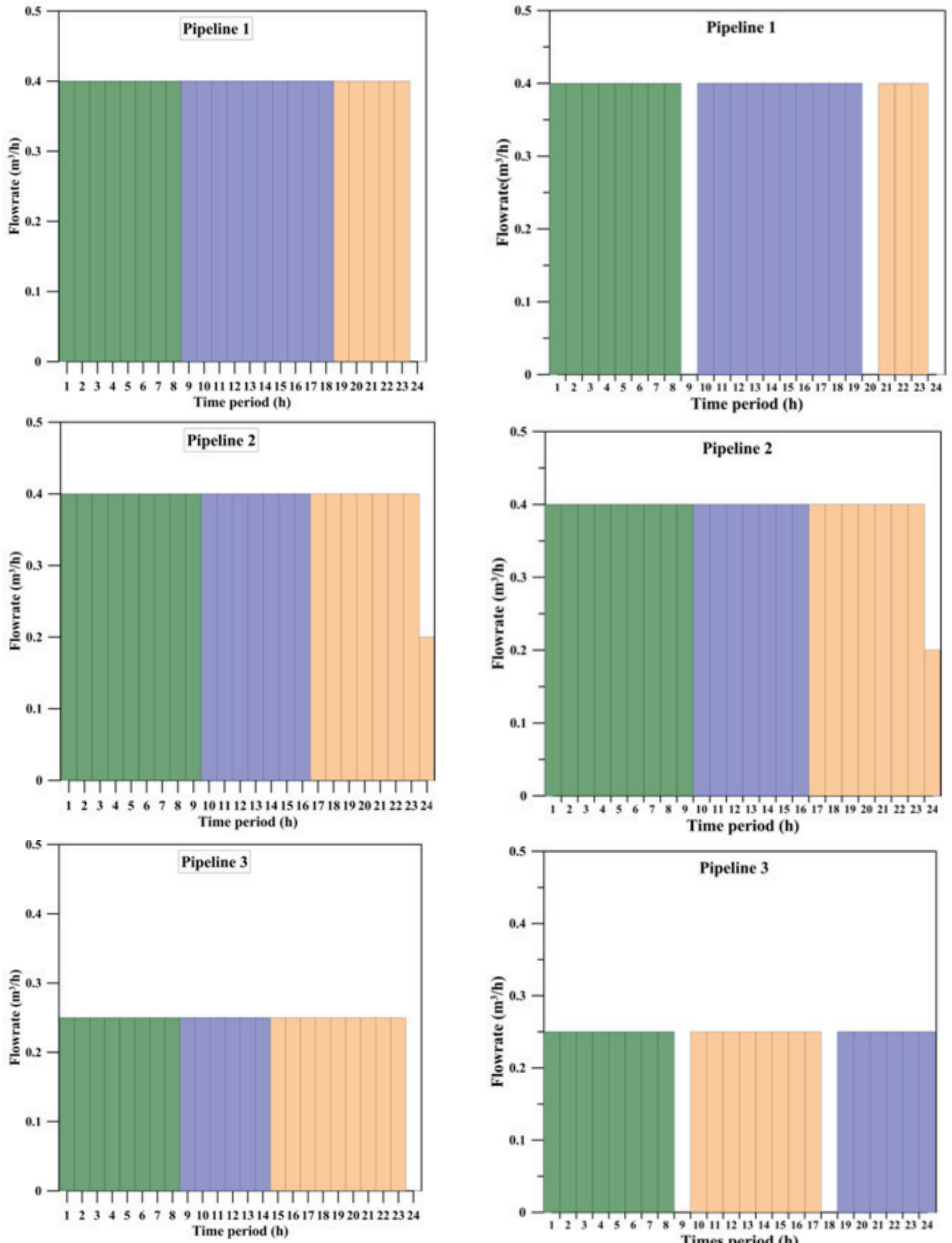

Figure 6. Pipeline flowrate profile (D1 - blue, D2 - green, D3 orange) - Base Model.

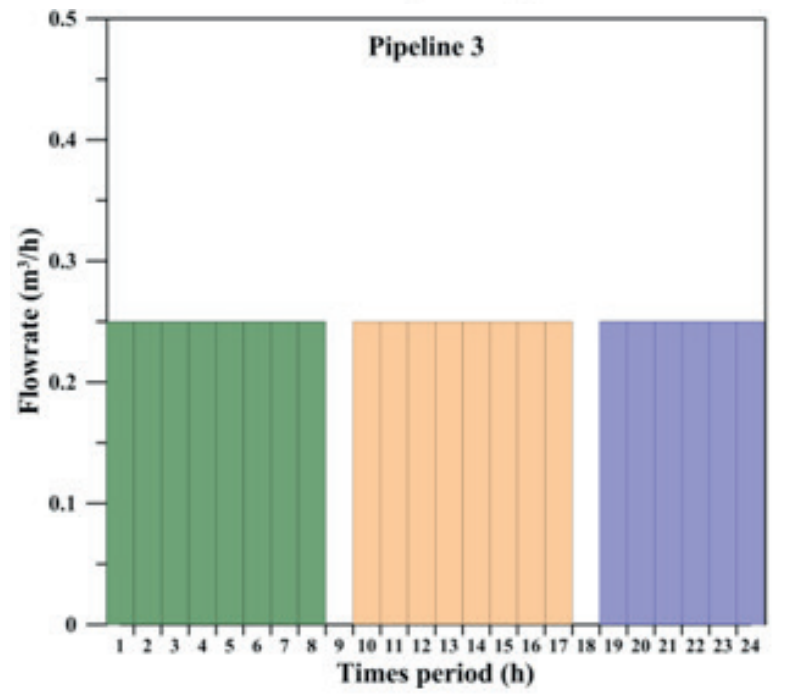

Figure 7. Pipeline flowrate profile (D1 - blue, D2 - green, D3 orange)-Model 1 . 
A Novel Transition Identification Mechanism for the Diesel Blending and Distribution
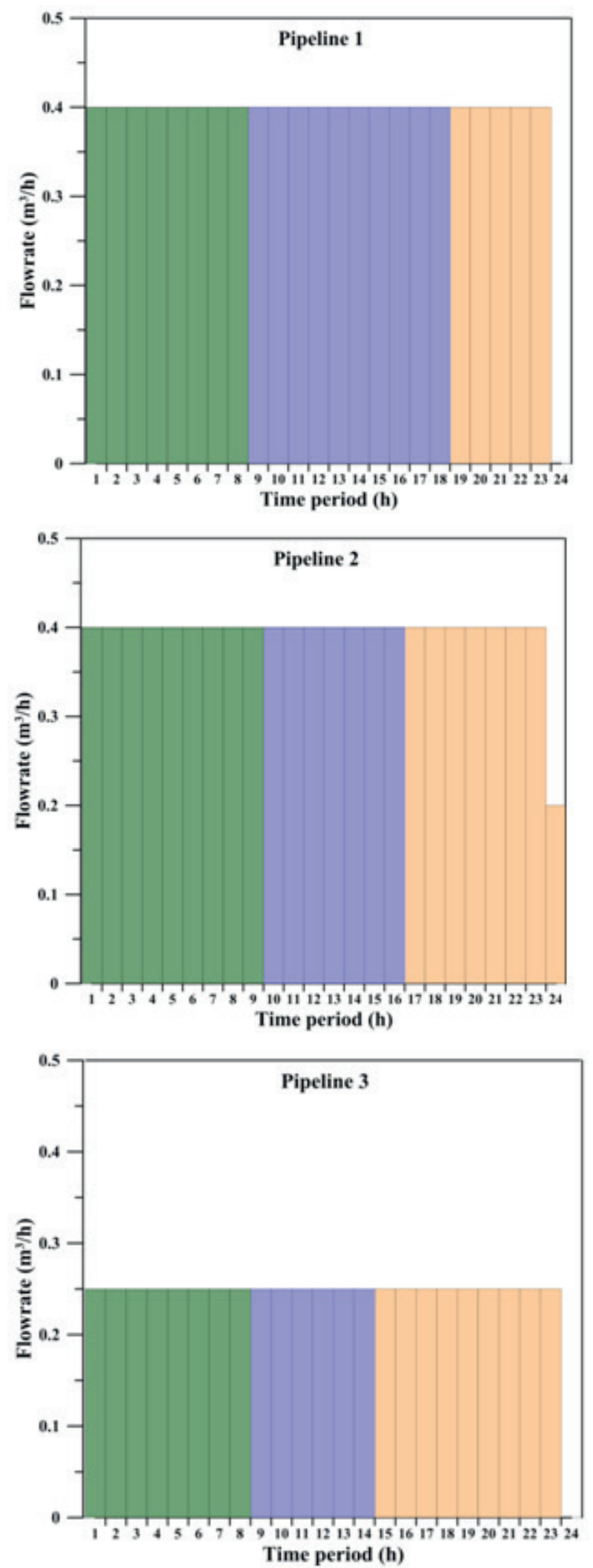

Figure 8. Pipeline flowrate profile (D1 - blue, D2 - green, D3 orange) - Model 2 .
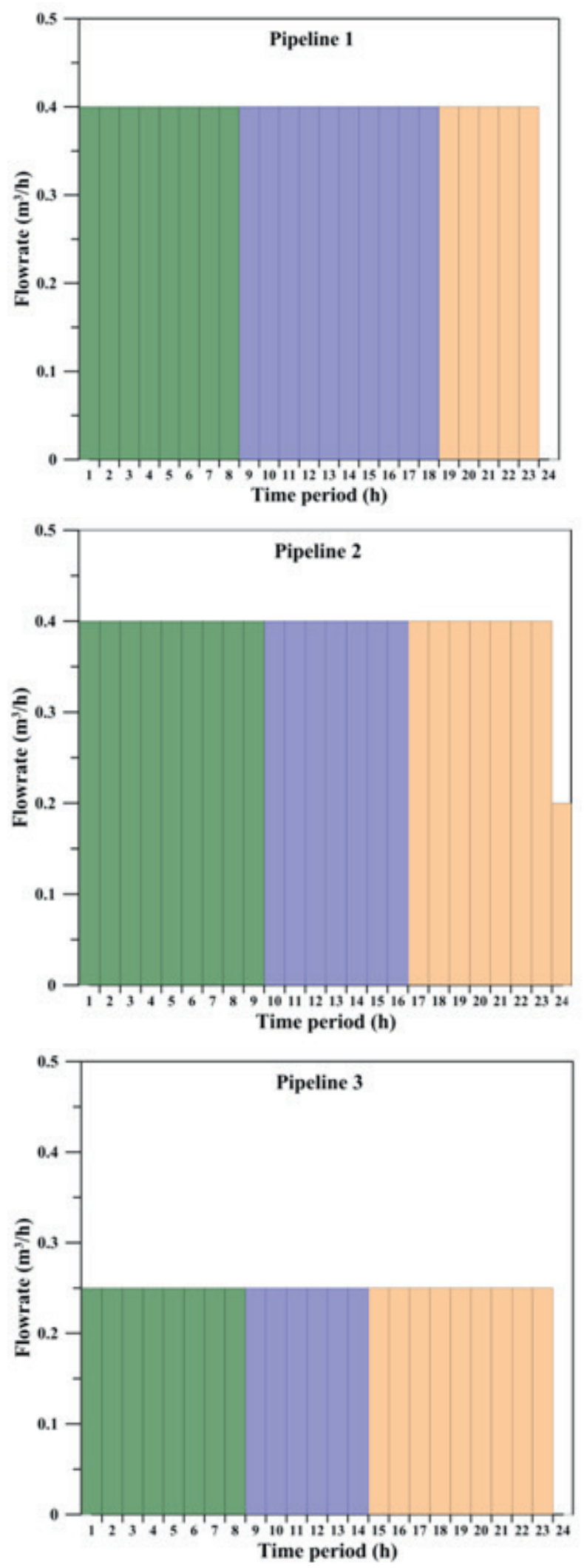

Figure 9. Pipeline flowrate profile (D1 - blue, D2 - green, D3 orange)-Model 3. 
(a)
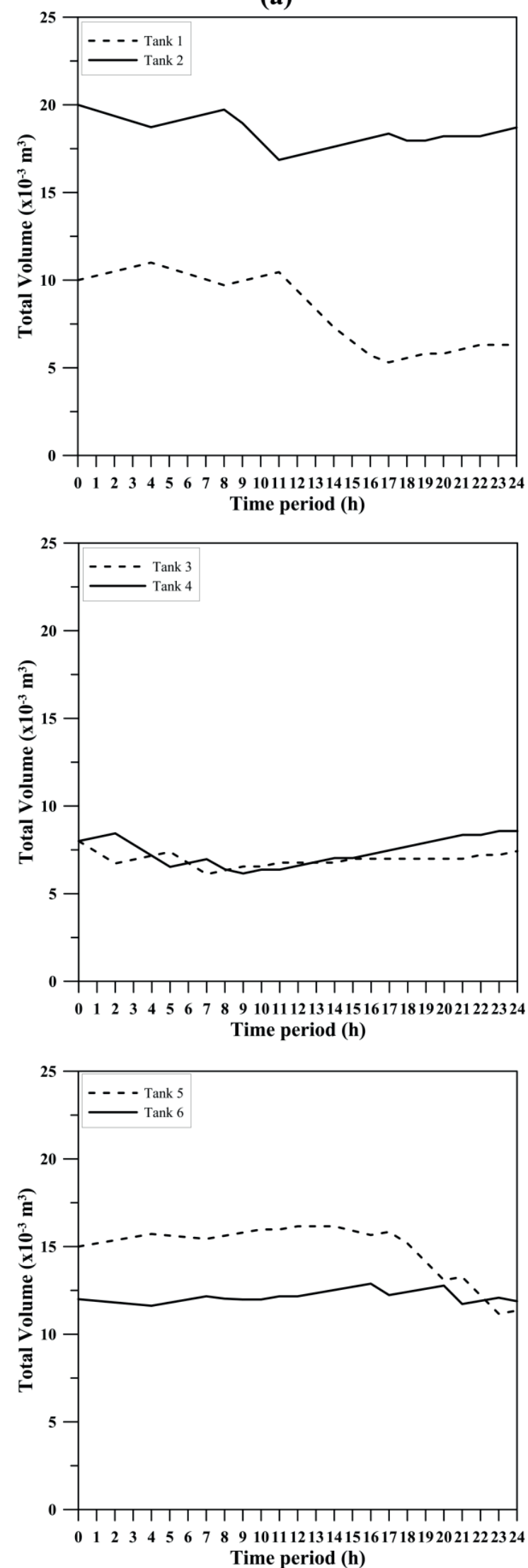

(b)
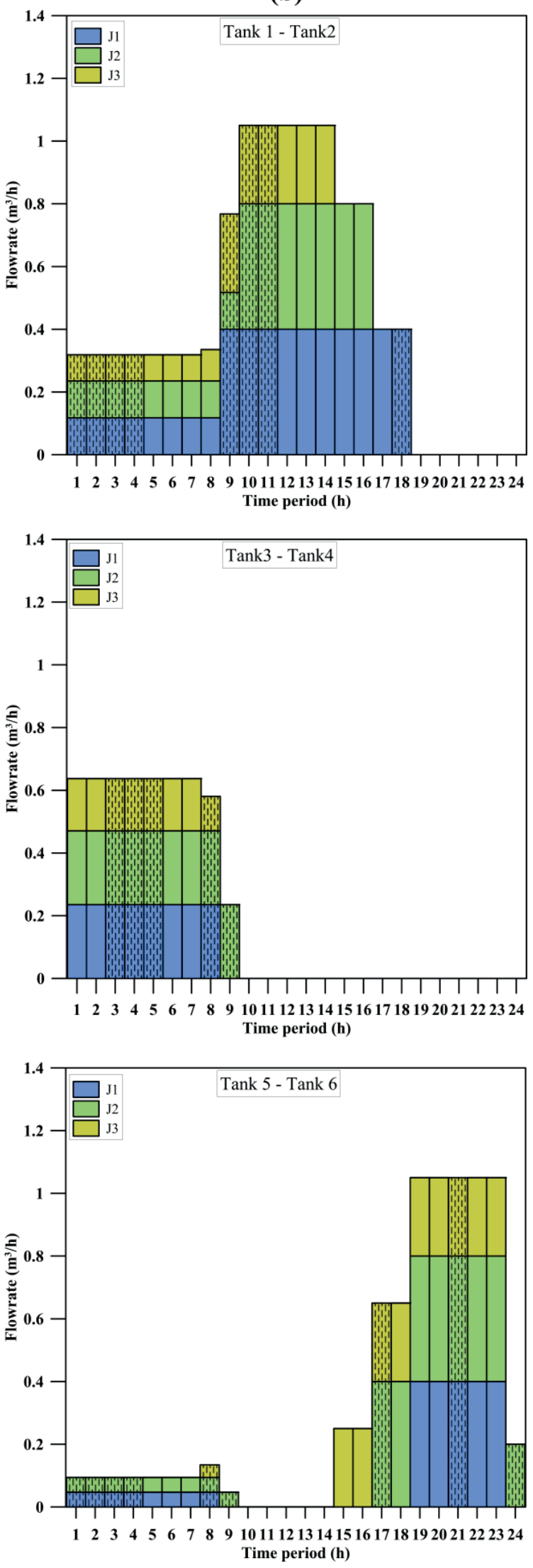

Figure 10. (a) Inventory profile and (b) Flowrate between tanks and pipelines (J1 - blue, J2 - green, J3 - yellow) - Base model. 
(a)
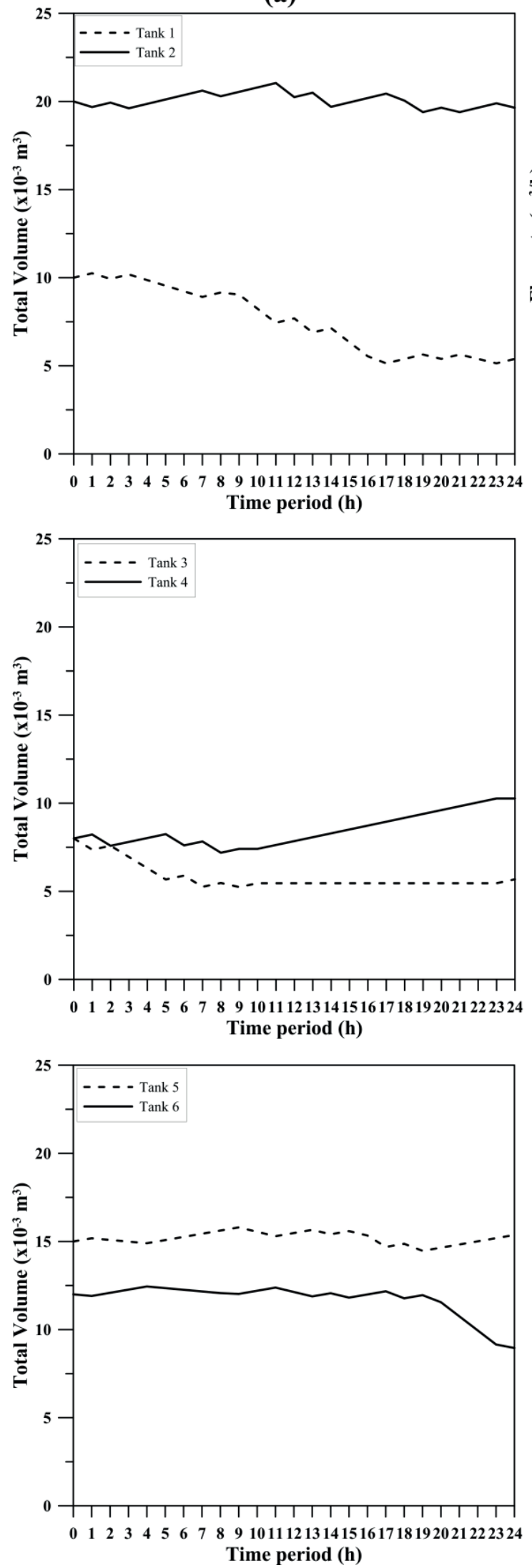

(b)
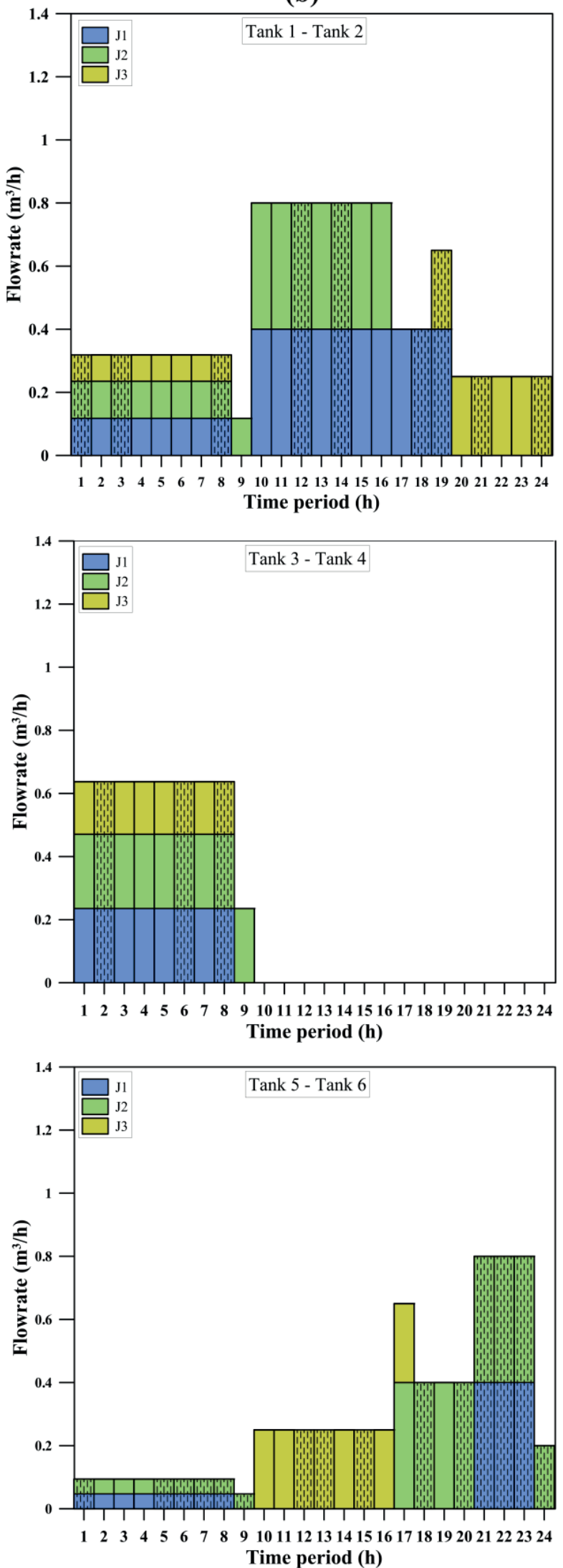

Figure 11. (a) Inventory profile and (b) Flowrate between tanks and pipelines (J1 - blue, J2 - green, J3 - yellow) - Model 1. 
(a)
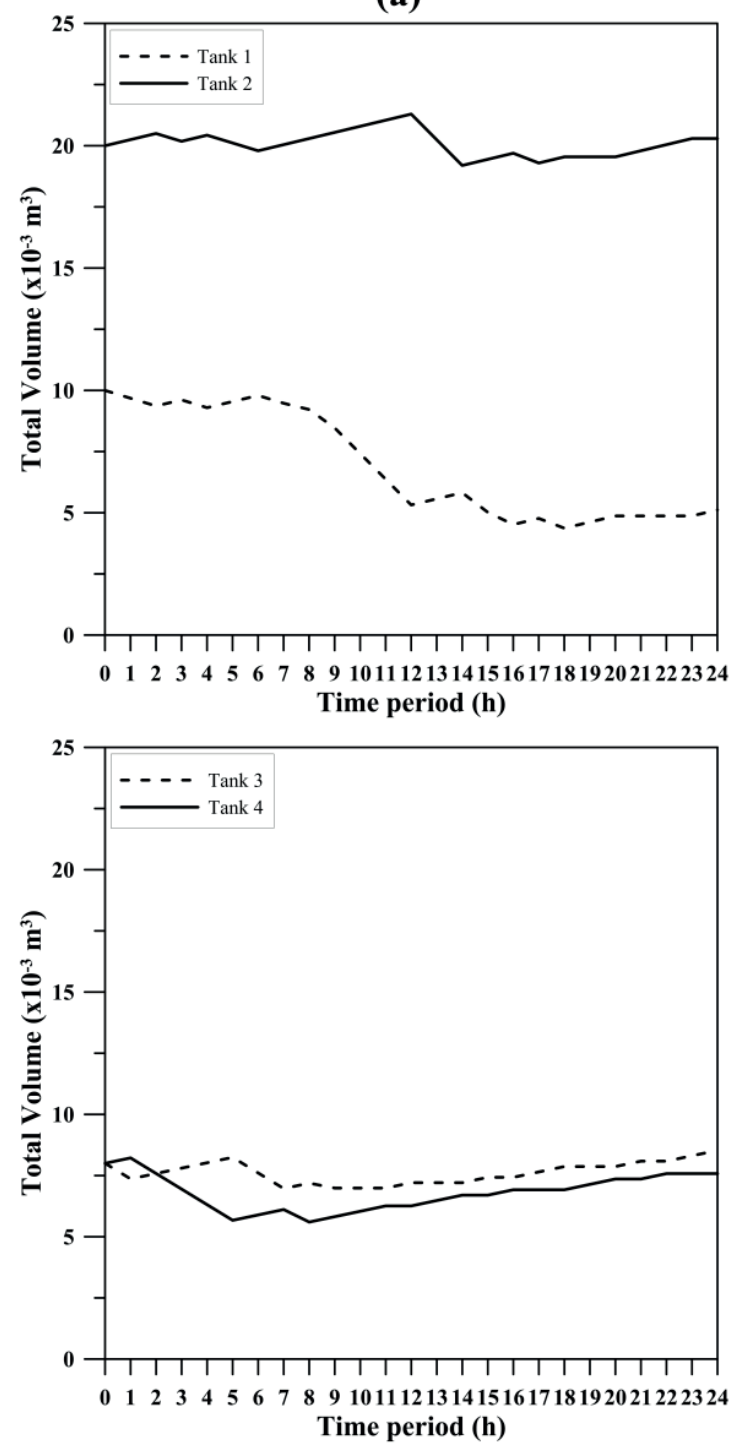

(b)
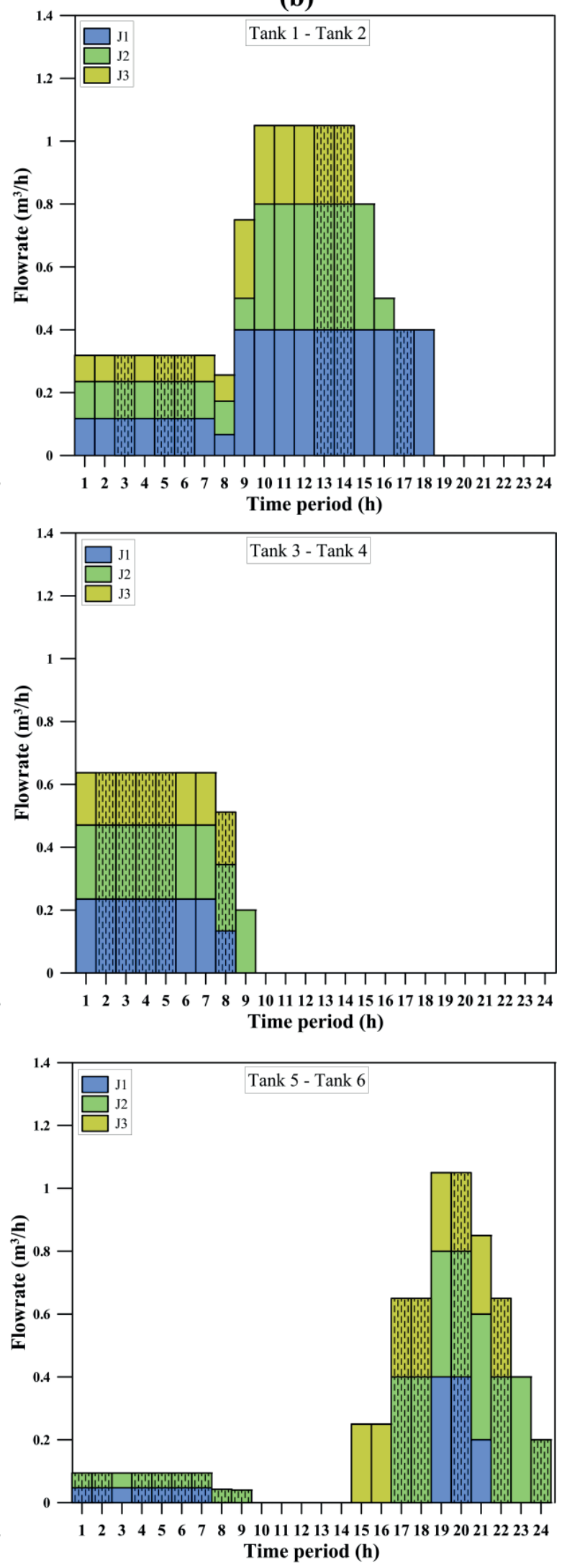

Figure 12. (a) Inventory profile and (b) Flowrate between tanks and pipelines (J1 - blue, J2 - green, J3 - yellow) - Model 2. 
A Novel Transition Identification Mechanism for the Diesel Blending and Distribution

(a)
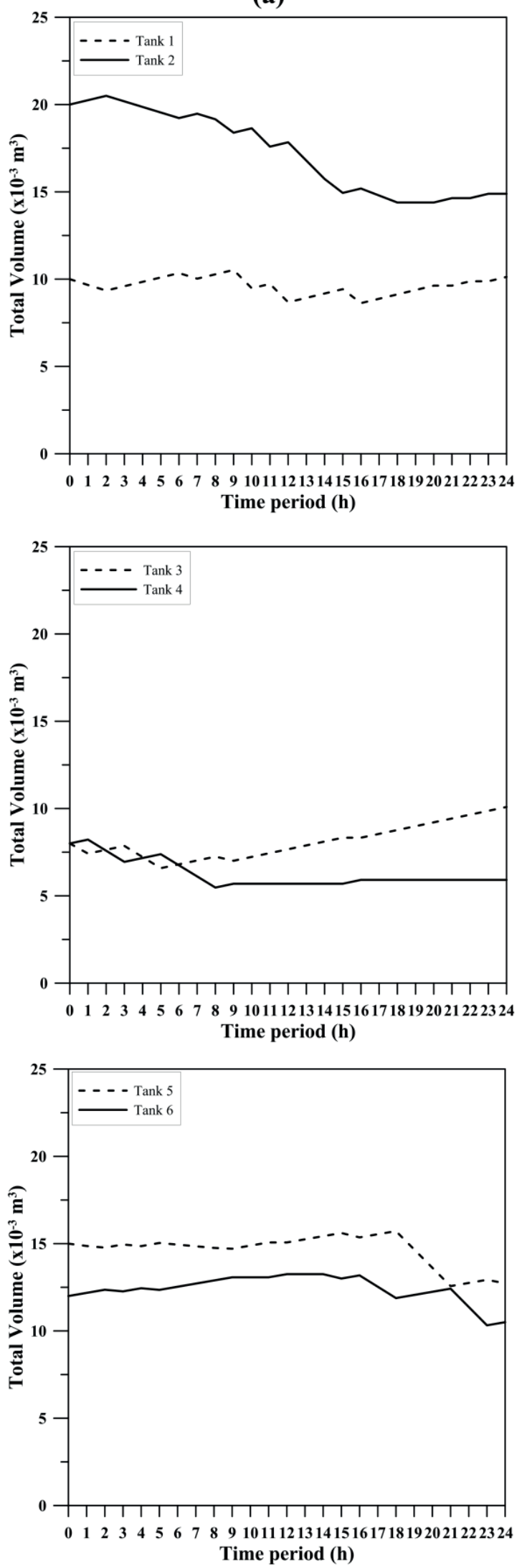

(b)
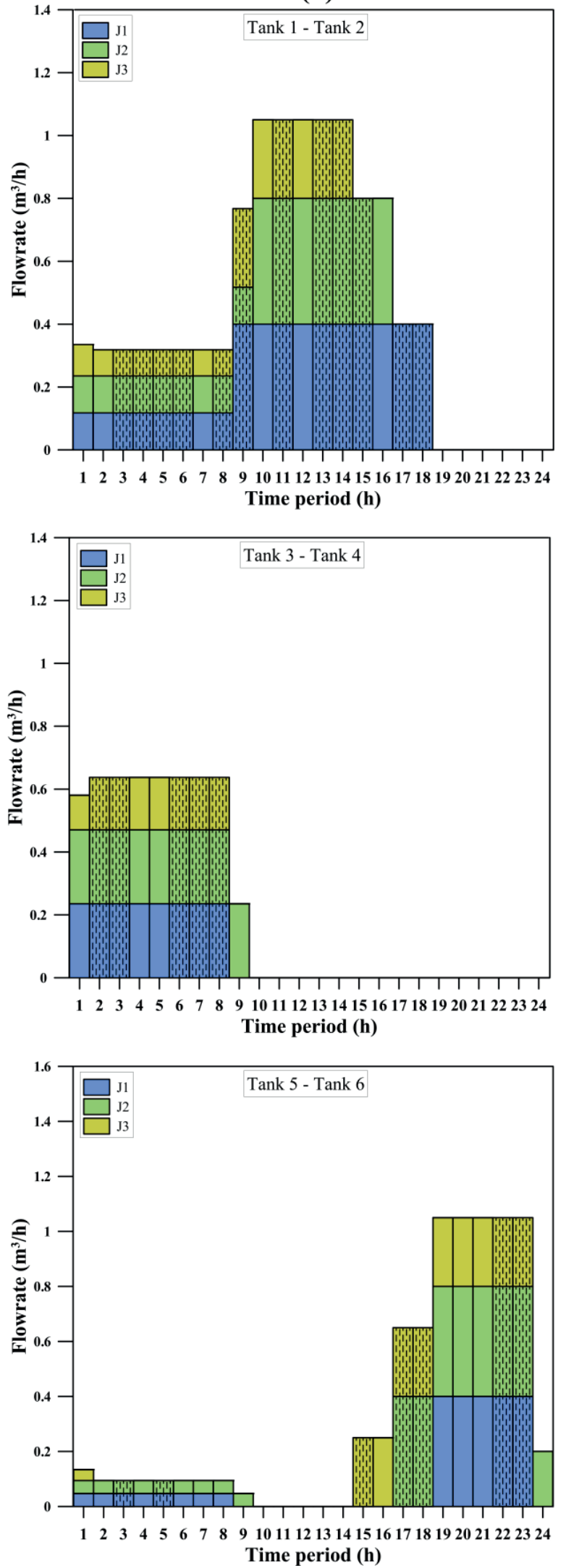

Figure 13. (a) Inventory profile and (b) Flowrate between tanks and pipelines (J1 - blue, J2 - green, J3 - yellow) - Model 3. 


\section{Long-Term Time Horizon}

Table 10 shows statistical data for the solution of model 4 for a time horizon comprising four days for two distinct scenarios: 1-) demand is exactly satisfied, and 2-) demand is to be over satisfied. For sake of comparison, the base model was also solved for each day sequentially, which means that the second day was optimized after decisions of the first day had been made (frozen); the third day was optimized after decisions of the first two days had been made, and so forth. The inventory of one day was taken as the starting inventory of the next one. Likewise, the first shipment allocated in a day took into consideration the last product dispatched in the previous day, so that the transition cost between different days would be properly accounted for.

Table 10. Computational statistics -Long-term time horizon.

\begin{tabular}{|c|c|c|c|c|}
\hline & \multicolumn{2}{|c|}{ Model 4} & \multicolumn{2}{|c|}{ Sequential Base Model } \\
\hline & $\begin{array}{r}\text { Demand } \\
\text { exactly } \\
\text { satisfied }\end{array}$ & $\begin{array}{r}\text { Demand } \\
\text { over } \\
\text { satisfied }\end{array}$ & $\begin{array}{l}\text { Demand } \\
\text { exactly } \\
\text { satisfied }\end{array}$ & $\begin{array}{r}\text { Demand } \\
\text { over } \\
\text { satisfied }\end{array}$ \\
\hline Equations & 12,392 & 12,392 & 12,560 & 12,560 \\
\hline Binary variables & 5,328 & 5,328 & 5,112 & 5,112 \\
\hline Continuous variables & 9,181 & 9,181 & 9,020 & 9,020 \\
\hline Objective function (\$) & $2,350.70$ & $2,318.56$ & $3,487.53$ & $2,746.88$ \\
\hline Best bound & 2348.37 & $2,316.99$ & $3,487.34$ & $2,746.65$ \\
\hline CPU time (s) & 314,86 & 516.83 & 77.14 & 102.80 \\
\hline Relative gap (\%) & 0.09 & 0.06 & 0.009 & 0.01 \\
\hline $\mathrm{B} \& \mathrm{~B}$ root solution & 637.10 & 579.73 & $--*$ & $--*$ \\
\hline Time for $1^{\text {st }}$ int. sol. (gap) & $112.49(44.47)$ & $16.24(64.76)$ & $--^{*}$ & $--*$ \\
\hline Nodes & 11,276 & 14,565 & 3,059 & 5,205 \\
\hline Iterations & $2,193,536$ & $2,368,720$ & 167,781 & 176,072 \\
\hline Material cost (\$) & 21.57 & 22.87 & 21.63 & 21.63 \\
\hline Pumping cost (\$) & 11.78 & 13.40 & 11.78 & 16.08 \\
\hline Inventory cost (\$) & 627.35 & 592.29 & 714.12 & 582.43 \\
\hline Transition cost $(\$)$ & $1,690.00$ & $1,690.00$ & $2,740.00$ & $2,120.00$ \\
\hline
\end{tabular}

* Since the sequential base model comprises the solution of the base model sequentially, there is not a unique value that represents this information.

It should be noted that the total number of constraints generated by model 4 is less than the total number of constraints generated by the sequential base model. On the other hand, the number of continuous and binary variables was higher for model 4 in comparison to the sequential base model. At first glance, one might think that the total number of variables and constraints of model 4 should be four times the total number of variables and constraints of model 3 plus the added constraints to deal with event points, since model 4 (solved for 4 days) derives from model 3 (solved for 1 day) and, therefore, the number of variables and constraints of model 4 should be greater than the sequential base model. However, it should be borne in mind that the transition constraints in model 4 are created only for event points (12 for each pipeline), not for time periods ( 96 for each pipeline) as is the case of the sequential base model.

A comparison of the two approaches in terms of the objective function and cost components reveals a substantial difference caused by transition cost followed by inventory cost, proving that it is very beneficial to produce a schedule looking far into the future. On the other hand, a price has to be paid. Model 4 spent much more time to find the optimal solution in both scenarios, which again can be attributed to the poor relaxation produced by the proposed approach. Note also that the problems can be solved in little less computational time when demand is exactly satisfied due to better relaxation. On the other hand, when demand is over satisfied there is an opportunity for reducing the number of transitions by pumping more of a product and thus reducing transition costs, which translates into savings. In fact, comparison of the results produced by model 4 and the sequential base model for scenario 2 accounts for savings on the order of $32 \%$.

Figures 14 and 15 present Gantt charts for model 4 and the sequential base model for scenarios 1 and 2, respectively. Comparing solutions produced by model 4 in the two pictures it can be observed that the same sequence was obtained with the two cases. The only difference is the amount of each product sent in each parcel. In contrast, solutions obtained with the sequential base model for the two cases differ in both sequence and shipped volumes. 
A Novel Transition Identification Mechanism for the Diesel Blending and Distribution Scheduling Problem Using the Discrete Time Representation with Two Time-Scales Granularity

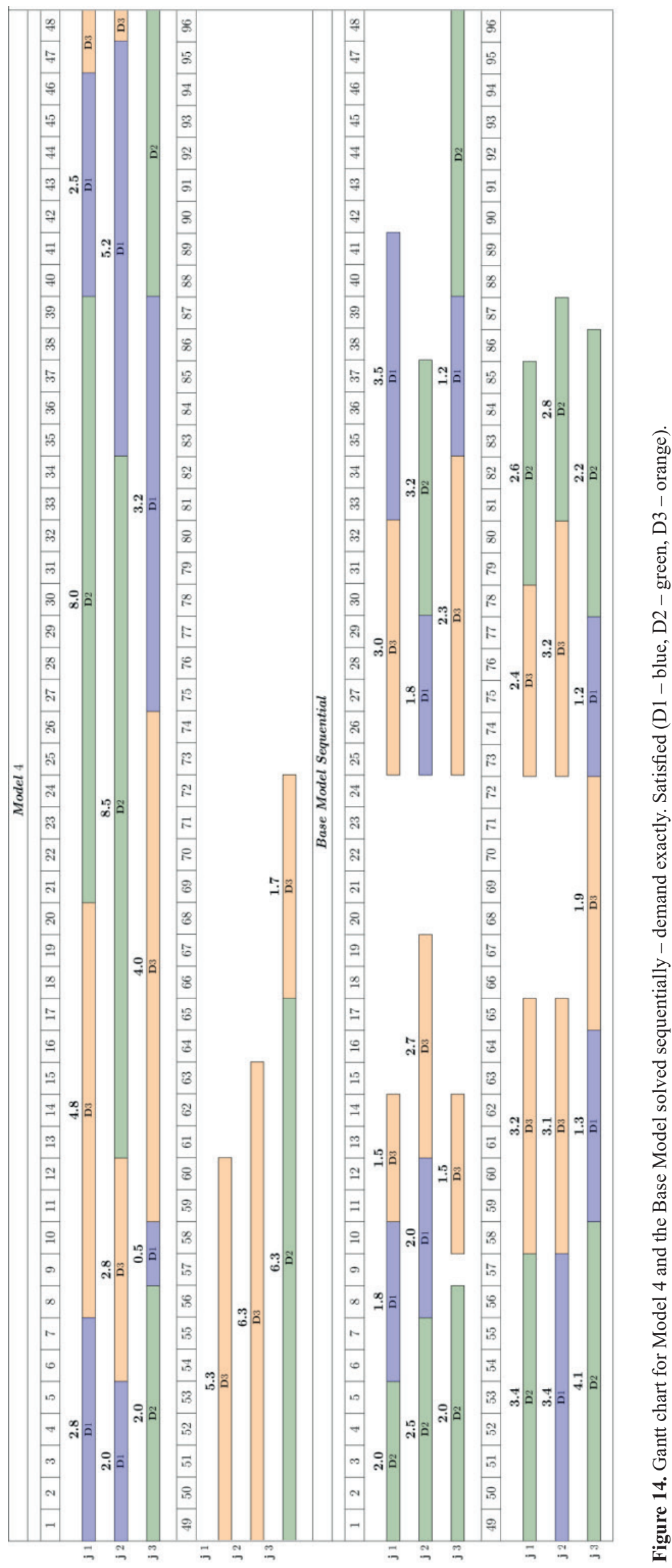




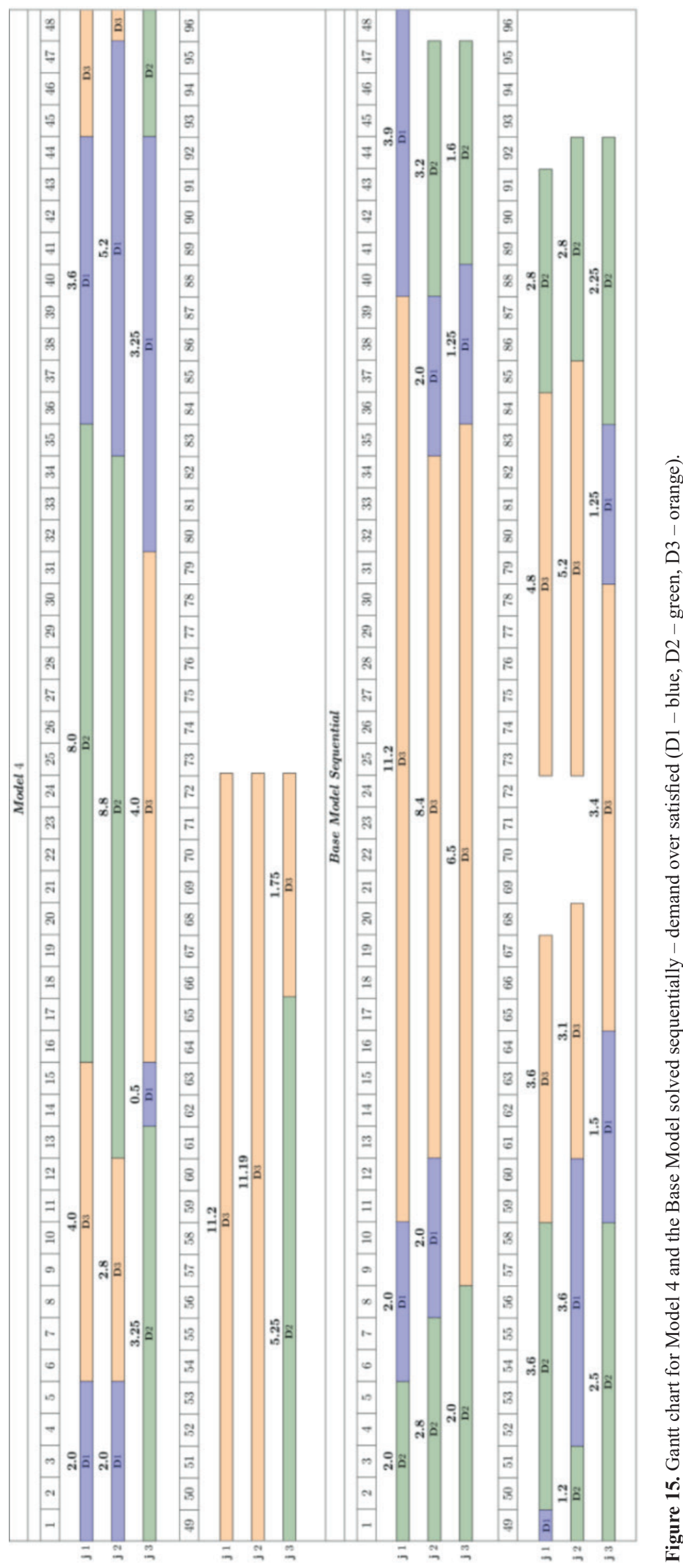




\section{CONCLUSIONS}

In this work, the blending and distribution problem in the context of the oil industry has been taken as the object of study to address different ways of treating transition identification in pipelines. The discrete time formulation originally proposed by Pinto et al. (2000) was taken as a starting point, which was originally developed for addressing short-term blending and distribution schedules. Alternative approaches were studied and a new approach was proposed to address transition identification with the intent of enabling addressing problems with longer time horizons. The proposed approach was considered the most promising, which was embodied in a fourth approach considering two levels of granularity for time periods. Event points were postulated so as to reduce the number of transition constraints, since model size increases very quickly with time horizon. Results demonstrated that the final proposed formulation offers real saving opportunities, although solution time might be a problem depending on the length of the time horizon considered. The assumption that the quality of the intermediate streams produced by distillation units is static is somewhat limiting in representing real world scenarios. Likewise, normally, blended materials are accumulated and kept in storage tanks for certification purposes. A research program considering continuous time formulation and the facts just described is underway and, hopefully, the results will be published in the near future.

\section{ACKNOWLEDGEMENTS}

The authors gratefully acknowledge financial support received from $\mathrm{CAPES}$.

\section{REFERENCES}

Boschetto, S. N., Magatão, L., Brondani, W. M., Neves-Jr, F., Arruda, L. V., Barbosa-Póvoa, A. P., and Relvas, S., An operational scheduling model to product distribution through a pipeline network. Industrial \& Engineering Chemistry Research, 49(12), 5661-5682 (2010).

Cafaro, D. C., and Cerdá, J., Optimal scheduling of multiproduct pipeline systems using a non-discrete MILP formulation. Computers and Chemical Engineering, 28, 2053-2068 (2004).

Cafaro, D. C, and Cerdá, J., Optimal scheduling of refined products pipelines with multiple sources. Industrial \& Engineering Chemistry Research, 48(14), 6675-6689 (2009).

Cafaro, V.G., Cafaro, D. C., Mendez, C. A., and Cerdá, J., MINLP model for the detailed scheduling of refined products pipelines with flow rate dependent pumping costs. Computers and Chemical Engineering, 72, 210-221 (2015).

Catillo-Castillo, P. A., and Mahalec, V., Improved continuous time model for gasoline blend scheduling. Computers and Chemical Engineering, 84, 627-646 (2016).
Chen X., Grossmann, I. E., and Zheng, L., A comparative study of continuous time models for scheduling of crude oil operations in inland refineries. Computers and Chemical Engineering, 44, 141-167 (2012).

Furman, K. C., Jia, Z., and Ierapetritou, M. G., A robust event-based continuous time formulation for tank transfer scheduling. Industrial \& Engineering Chemistry Research, 46(26), 9126-9136 (2007).

Gao, X., Jiang, Y., Chen, T., and Huang, D., Optimizing scheduling of refinery operations based on piecewise linear models. Computers and Chemical Engineering, 75, 105-119 (2015).

Ghaffari-Hadigheh, A., and Mostafaei, H., On the scheduling of real world multiproduct pipelines with simultaneous delivery. Optimization and Engineering, Springer, 16(3), 571-604, (2014).

Glismann, K., and Gruhn, G., Short-term scheduling and recipe optimization of blending processes. Computers and Chemical Engineering, 25, 627-634 (2001).

Goethe-Lundgren, M., Lundgren, J. T., and Persson, J. A., An optimization model for refinery production scheduling. International Journal Production Economics 78, 255-270 (2002)

Harjunkoski, I., Maravelias, C. T., Bongers, P., Castro, P. M., Engell, S., Grossmann, I. E., Hooker, J., Mendez, C., Guido S., and Wassick J., Scope for industrial applications of production scheduling models and solution methods. Computers and Chemical Engineering. 62, 161-193 (2014).

Jia, Z., and Ierapetritou, M. G., Mixed-Integer Linear Programming Model for Gasoline Blending and Distribution Scheduling. Industrial \& Engineering Chemistry Research, 42(4), 825-835 (2003).

Jia, Z., and Ierapetritou, M. G., Efficient short-term scheduling of refinery operations based on a continuous time formulation. Computers and Chemical Engineering, 28, 1001-1019 (2004).

Jia, Z., Ierapetritou, M. G., and Kelly, J. D., Refinery Short-Term Scheduling Using Continuous Time Formulation: Crude-Oil Operations, Industrial \& Engineering Chemistry Research 42(13), 3085-3097 (2003).

Karamarkar, U.S., and Schrage, L., The deterministic dynamic product cycling problem. Operations Research, 33, 326 -345 (1985).

Kondili, E., Pantelides, C. C., and Sargent, R. W. H., A general algorithm for short term scheduling of batch operations - I MILP formulation. Computers and Chemical Engineering, 17(2), 211-227 (1993).

Lee, H., Pinto, J. M., Grossmann, I. E., and Park, S., MixedInteger Linear Programming Model for Refinery ShortTerm Scheduling of Crude Oil Unloading with Inventory Management. Industrial and Engineering Chemistry Research, 35(5), 1630-1641 (1996).

Li J., and Karimi I. A., Scheduling gasoline blending operations from recipe determination to shipping using unit slots. Industrial \& Engineering Chemistry Research, 50, 9156-9174 (2011).

Li, J., Karimi, I. A., and Srinivasan, R., Recipe determination and scheduling of gasoline blending operations. AIChE, 56(2), 441-465 (2010). 
Liu, S., Pinto, J. M., and Papageorgiou, L. G., MILP-based approaches for medium-term planning of single-stage continuous multiproduct plants with parallel units. Computers Management Science, 7, 407-435 (2010).

Luo, C., and Rong, G., Hierarchical approach for short-term scheduling in refineries. Industrial \& Engineering Chemistry Research, 46, 3656-3668 (2007).

Mendez, C. A., Grossmann, I. E., Harjunkoski, I., and Kabore, P., A simultaneous optimization approach for off-line blending and scheduling of oil-refinery operations. Computers and Chemical Engineering, 30, 614-634 (2006).

Mendez, C. A., Cerdá, J., Grossmann, I. E., Harjunkoski, I., and Fahl, M., State-of-the-art review of optimization methods for short-term scheduling of batch processes. Computers and Chemical Engineering, 30, 913-946 (2006).

MirHassani, S. A., and BeheshtiAsl, N., A heuristic batch sequencing for multiproduct pipelines. Computers and Chemical Engineering, 56, 58-67 (2013).

Moro, L. F. L., and Pinto, J. M., Mixed-Integer Programming Approach for Short-Term Crude Oil Scheduling, Industrial \& Engineering Chemistry Research, 43(1), 85-94 (2004).

Mouret, S., Grossmann, I. E., and Pestiaux, P., A novel priorityslot based continuous-time formulation for crude oil scheduling problems. Industrial \& Engineering Chemistry Research, 48(18), 8515-8528 (2009).

Pinto, J. M., Joly M., and Moro, L. F. L., Planning and scheduling models for refinery operations. Computers and Chemical Engineering, 24, 2259-2276 (2000).

Rejowski Jr., R., and Pinto, J. M., Scheduling of a multiproduct pipeline system. Computers and Chemical Engineering, 27, 1229-1246 (2003).
Rejowsi Jr., R., and Pinto, J. M., Efficient MILP formulations and valid cuts formultion product pipeline scheduling. Computers and Chemical Engineering, 28, 1511-1528 (2004).

Relvas, S., Matos, H. A., Barbosa-Póvoa, A. P. F. D., and Fialho, J., Reactive scheduling framework form a multiproduct pipeline with inventory management. Industrial and Engineering Chemistry Research, 46(17), 5659-5672 (2007).

Rigby, B., Lasdon, L. S., and Waren, A. D. (1995). The evolution of texaco's blending systems: from OMEGA to StarBlend. Interfaces, 25(5), 64-83.

Saharidis, G. K. D., and Ierapetritou, M. G., Scheduling of loading and unloading of crude oil in a refinery with optimal mixture preparation. Industrial \& Engineering Chemistry Research, 48(5), 2624-2633 (2009).

Sahinidis, N.V., and Grossmann, I.E., MINLP model for cyclic multiproduct scheduling on continuous parallel lines. Computers and Chemical Engineering, 15(2), 85-103 (1991).

Shah, N. K., and Ierapetritou, M., Lagrangian decomposition approach to scheduling large-scale refinery operations. Computers and Chemical Engineering, 79, 1-29 (2015).

Shah, N., Saharidis, G. K. D., Jia, Z., and Ierapetritou, M. G., Centralized-decentralized optimization for refinery scheduling. Computers and Chemical Engineering, 33, 20912105 (2009).

Simão, L. M., Dias, D. M., and Pacheco, M. A. C., Refinery Scheduling Optimization using Genetic Algorithms and Cooperative Coevolution. Proceedings of the 2007 IEEE Symposium on Computational Intelligence in Scheduling, 151-158 (2007).

Wolsey, L.A., MIP modeling of change-over in production planning and scheduling problems. European Journal of Operational Research, 99, 154-165 (1997). 


\section{APPENDIX}

\section{A.1 - Complete Model 1}

Objective function

$$
\min \sum_{i \in I} \sum_{j \in J} \sum_{t \in T}\left(C r m_{i}+C p_{i}\right) F_{i, j, t}+\sum_{i \in I} \sum_{t \in T} C i_{i} V_{i, t}+\sum_{j \in J} \sum_{p \in P} \sum_{n \in P, p \neq n} C t_{p, n} W_{j, p, n}
$$

Material balance constraints

$$
\begin{gathered}
V_{i, t}=V_{i}^{0}+\sum_{t^{\prime} \leq t}\left(F_{i, t}-\sum_{j \in J_{i}} F_{i, j, t}\right) \forall i \in I, t \in T \\
V_{i}^{\min } \leq V_{i, t} \leq V_{i}^{\max } \forall i \in I, t \in T \\
\sum_{p \in P} F_{j, p, t}=\sum_{i \in I_{j}} F_{i, j, t} \forall j \in J, t \in T \\
\sum_{p \in P} x_{p, k}^{s p c} F_{j, p, t} \geq \sum_{i \in I} x_{i, k}^{0} F_{i, j, t} \forall j \in J, k=1, t \in T \\
\sum_{p \in P} x_{p, k}^{s p c} F_{j, p, t} \leq \sum_{i \in I} x_{i, k}^{0} F_{i, j, t} \quad \forall j \in J, k=2, t \in T .
\end{gathered}
$$

Demand constraint

$$
\sum_{t \in T} F_{j, p, t} \geq D_{j, p} \forall j \in J, p \in P
$$

Operating rules and logic constraints

$$
\begin{gathered}
\sum_{i \in I_{u}} X_{i, t}=1 \forall u \in D U, t \in T \\
\sum_{t \in T} Z_{j, p, t}^{S} \leq 1 \forall j \in J, p \in P \\
\sum_{t \in T}\left(Z_{j, p, t}^{S}-Z_{j, p, t}^{E}\right)=0 \forall j \in J, p \in P \\
T_{j, p}^{S}=\sum_{t \in T} t \cdot Z_{j, p, t}^{S} \forall j \in J, p \in P \\
T_{j, p}^{E}=\sum_{t \in T} t . Z_{j, p, t}^{E} \forall j \in J, p \in P \\
T_{j, p}^{S} \leq T_{j, p}^{E} \forall j \in J, p \in P \\
Z_{j, p, t}=\sum_{t^{\prime} \leq t} Z_{j, p, t^{\prime}}^{S}-\sum_{t^{\prime}<t} Z_{j, p, t^{\prime}}^{E} \forall j \in J, p \in P, t \in T
\end{gathered}
$$




$$
\begin{gathered}
N C_{i} X_{i, t}+\sum_{j \in J_{i}} Y_{i, j, t} \leq N C_{i} \forall i \in I, t \in T \\
Y_{i, j, t} \leq \sum_{p \in P} Z_{j, p, t} \forall i \in I, j \in J, t \in T \\
\sum_{p \in P} Z_{j, p, t} \leq 1 \forall j \in J, t \in T
\end{gathered}
$$

Flowrate constraints

Transition constraints

$$
\begin{gathered}
F_{i}^{\text {min }} X_{i, t} \leq F_{i, t} \leq F_{i}^{\max } X_{i, t} \quad \forall i \in I, t \in T \\
F_{i, j}^{\min } Y_{i, j, t} \leq F_{i, j, t} \leq F_{i, j}^{\max } Y_{i, j, t} \quad \forall i \in I, j \in J, t \in T \\
F_{j}^{\min } Z_{j, p, t} \leq F_{j, p, t} \leq F_{j}^{\max } Z_{j, p, t} \quad \forall i \in I, p \in P, t \in T
\end{gathered}
$$

$$
W_{j, p, n} \geq Z_{j, p, t}+Z_{j, n, t+1}-1 \forall j \in J, \quad p, n \in P, t \in T, p \neq n
$$

\section{A.2 - Complete Model 2}

Objective function

$$
\min \sum_{i \in I} \sum_{j \in J} \sum_{t \in T}\left(C r m_{i}+C p_{i}\right) F_{i, j, t}+\sum_{i \in I} \sum_{t \in T} C i_{i} V_{i, t}+\sum_{j \in J} \sum_{p \in P} \sum_{n \in P, p \neq n} C t_{p, n} W_{j, p, n}
$$

Material balance constraints

$$
\begin{gathered}
V_{i, t}=V_{i}^{0}+\sum_{t^{\prime} \leq t}\left(F_{i, t}-\sum_{j \in J_{i}} F_{i, j, t}\right) \quad \forall i \in I, t \in T \\
V_{i}^{\min } \leq V_{i, t} \leq V_{i}^{\max } \quad \forall i \in I, t \in T \\
\sum_{p \in P} F_{j, p, t}=\sum_{i \in I_{j}} F_{i, j, t} \quad \forall j \in J, t \in T \\
\sum_{p \in P} x_{p, k}^{s p c} F_{j, p, t} \geq \sum_{i \in I} x_{i, k}^{0} F_{i, j, t} \quad \forall j \in J, k=1, t \in T \\
\sum_{p \in P} x_{p, k}^{s p c} F_{j, p, t} \leq \sum_{i \in I} x_{i, k}^{0} F_{i, j, t} \quad \forall j \in J, k=2, t \in T
\end{gathered}
$$


Demand constraint

$$
\sum_{t \in T} F_{j, p, t} \geq D_{j, p} \quad \forall j \in J, p \in P
$$

Operating rules and logic constraints

$$
\begin{gathered}
\sum_{i \in I_{u}} X_{i, t}=1 \quad \forall u \in D U, t \in T \\
\sum_{t \in T} Z_{j, p, t}^{S} \leq 1 \quad \forall j \in J, p \in P \\
\sum_{t \in T}\left(Z_{j, p, t}^{S}-Z_{j, p, t}^{E}\right)=0 \quad \forall j \in J, p \in P \\
T_{j, p}^{S}=\sum_{t \in T} t \cdot Z_{j, p, t}^{S} \quad \forall j \in J, p \in P \\
T_{j, p}^{E}=\sum_{t \in T} t \cdot Z_{j, p, t}^{E} \quad \forall j \in J, p \in P \\
T_{j, p}^{S} \leq T_{j, p}^{E} \quad \forall j \in J, p \in P \\
Z_{j, p, t}=\sum_{t^{\prime} \leq t} Z_{j, p, t^{\prime}}^{S}-\sum_{t^{\prime}<t} Z_{j, p, t^{\prime}}^{E} \quad \forall j \in J, p \in P, t \in T \\
N C_{i} X_{i, t}+\sum_{j \in J_{i}} Y_{i, j, t} \leq N C_{i} \quad \forall i \in I, t \in T \\
Y_{i, j, t} \leq \sum_{p \in P} Z_{j, p, t} \quad \forall i \in I, j \in J, t \in T \\
\sum_{p \in P} Z_{j, p, t} \leq 1 \quad \forall j \in J, t \in T \\
\end{gathered}
$$

Flowrate constraints

$$
\begin{gathered}
F_{i}^{m i n} X_{i, t} \leq F_{i, t} \leq F_{i}^{\max } X_{i, t} \forall i \in I, t \in T \\
F_{i, j}^{\min } Y_{i, j, t} \leq F_{i, j, t} \leq F_{i, j}^{\max } Y_{i, j, t} \forall i \in I, j \in J, t \in T \\
F_{j}^{\min } Z_{j, p, t} \leq F_{j, p, t} \leq F_{j}^{\max } Z_{j, p, t} \forall i \in I, p \in P, t \in T
\end{gathered}
$$

Transition constraints

$$
W_{j, p, n, t} \geq Z_{j, p, t}+Z_{j, n, t^{\prime}}-\sum_{p^{\prime} \in P t^{\prime \prime}=t+1} \sum_{t^{\prime}-1} Z_{j, p^{\prime}, t^{\prime \prime}}-1 \forall j \in J, p, n \in P, t \in T, p \neq n
$$




\section{A.3 - Complete Model 3}

Objective function

$$
\min \sum_{i \in I} \sum_{j \in J} \sum_{t \in T}\left(C r m_{i}+C p_{i}\right) F_{i, j, t}+\sum_{i \in I} \sum_{t \in T} C i_{i} V_{i, t}+\sum_{j \in J} \sum_{p \in P} \sum_{n \in P, p \neq n} C t_{p, n} W_{j, p, n}
$$

Material balance constraints

$$
\begin{gathered}
V_{i, t}=V_{i}^{0}+\sum_{t^{\prime} \leq t}\left(F_{i, t}-\sum_{j \in J_{i}} F_{i, j, t}\right) \forall i \in I, t \in T \\
V_{i}^{\text {min }} \leq V_{i, t} \leq V_{i}^{\max } \forall i \in I, t \in T \\
\sum_{p \in P} F_{j, p, t}=\sum_{i \in I_{j}} F_{i, j, t} \forall j \in J, t \in T \\
\sum_{p \in P} x_{p, k}^{s p c} F_{j, p, t} \geq \sum_{i \in I} x_{i, k}^{0} F_{i, j, t} \forall j \in J, k=1, t \in T \\
\sum_{p \in P} x_{p, k}^{s p c} F_{j, p, t} \leq \sum_{i \in I} x_{i, k}^{0} F_{i, j, t} \forall j \in J, k=2, t \in T
\end{gathered}
$$

Demand constraint

$$
\sum_{t \in T} F_{j, p, t} \geq D_{j, p} \forall j \in J, p \in P
$$

Operating rules and logic constraints

$$
\begin{gathered}
\sum_{i \in I_{u}} X_{i, t}=1 \forall u \in D U, t \in T \\
\sum_{t \in T} Z_{j, p, t}^{S} \leq 1 \forall j \in J, p \in P \\
\sum_{t \in T}\left(Z_{j, p, t}^{S}-Z_{j, p, t}^{E}\right)=0 \forall j \in J, p \in P \\
T_{j, p}^{S}=\sum_{t \in T} t \cdot Z_{j, p, t}^{S} \forall j \in J, p \in P \\
T_{j, p}^{E}=\sum_{t \in T} t \cdot Z_{j, p, t}^{E} \forall j \in J, p \in P \\
T_{j, p}^{S} \leq T_{j, p}^{E} \forall j \in J, p \in P \\
Z_{j, p, t}=\sum_{t^{\prime} \leq t} Z_{j, p, t^{\prime}}^{S}-\sum_{t^{\prime}<t} Z_{j, p, t^{\prime}}^{E} \forall j \in J, p \in P, t \in T
\end{gathered}
$$




$$
\begin{gathered}
N C_{i} X_{i, t}+\sum_{j \in J_{i}} Y_{i, j, t} \leq N C_{i} \forall i \in I, t \in T \\
Y_{i, j, t} \leq \sum_{p \in P} Z_{j, p, t} \forall i \in I, j \in J, t \in T \\
\sum_{p \in P} Z_{j, p, t} \leq 1 \quad \forall j \in J, t \in T
\end{gathered}
$$

Flowrate constraints

$$
\begin{gathered}
F_{i}^{\min } X_{i, t} \leq F_{i, t} \leq F_{i}^{\max } X_{i, t} \forall i \in I, t \in T \\
F_{i, j}^{\min } Y_{i, j, t} \leq F_{i, j, t} \leq F_{i, j}^{\max } Y_{i, j, t} \forall i \in I, j \in J, t \in T \\
F_{j}^{\min } Z_{j, p, t} \leq F_{j, p, t} \leq F_{j}^{\max } Z_{j, p, t} \forall i \in I, p \in P, t \in T
\end{gathered}
$$

Transition constraints

$$
\begin{gathered}
\sum_{p \in P} Z_{j, p, t}+\sum_{p \in P} S_{j, p, t}=1 \forall j \in J, t \in T \\
\sum_{n \in P} Z_{j, n, t+1}+S_{j, p, t+1} \geq Z_{j, p, t}+S_{j, p, t} \forall j \in J, p \in P, t \in T-\{\mathrm{T}\} \\
W_{j, p, n} \geq Z_{j, p, t}+S_{j, p, t}+Z_{j, n, t+1}-1 \forall j \in J, p, n \in P, t \in T-\{\mathrm{T}\}, p \neq n
\end{gathered}
$$

\section{A.4 - Complete Model 4}

Objective function

$$
\min \sum_{i \in I} \sum_{j \in J} \sum_{t \in T}\left(C r m_{i}+C p_{i}\right) F_{i, j, t}+\sum_{i \in I} \sum_{t \in T} C i_{i} V_{i, t}+\sum_{j \in J} \sum_{p \in P} \sum_{n \in P, p \neq n \in \in E} \sum_{p, n} t_{j, p, n, e}
$$

Material balance constraints

$$
\begin{gathered}
V_{i, t}=V_{i}^{0}+\sum_{t^{\prime} \leq t}\left(F_{i, t^{\prime}}-\sum_{j \in J_{i}} F_{i, j, t^{\prime}}\right) \forall i \in I, t \in T \\
V_{i}^{\min } \leq V_{i, t} \leq V_{i}^{\max } \forall i \in I, t \in T \\
\sum_{p \in P} F_{j, p, t}=\sum_{i \in I_{j}} F_{i, j, t} \forall j \in J, t \in T
\end{gathered}
$$




$$
\begin{aligned}
& \sum_{p \in P} x_{p, k}^{s p c} F_{j, p, t} \geq \sum_{i \in I} x_{i, k}^{0} F_{i, j, t} \forall j \in J, k=1, t \in T \\
& \sum_{p \in P} x_{p, k}^{s p c} F_{j, p, t} \leq \sum_{i \in I} x_{i, k}^{0} F_{i, j, t} \quad \forall j \in J, k=2, t \in T
\end{aligned}
$$

Demand constraint

$$
\begin{gathered}
\sum_{t^{\prime} \leq t} F_{j, p, t^{\prime}} \geq \sum_{t^{\prime \prime} \leq t} D_{j, p, t^{\prime \prime}} \forall j \in J, p \in P, t, t^{\prime}, t^{\prime \prime} \in T, N T_{j}>1 \\
\sum_{t} F_{j, p, t} \geq \sum_{t} D_{j, p, t} \forall j \in J, p \in P, t \in T
\end{gathered}
$$

Operating rules and logic constraints

$$
\begin{gathered}
\sum_{i \in I_{u}} X_{i, t}=1 \forall u \in D U, t \in T \\
\sum_{t \in T_{d}} Z_{j, p, t, d}^{S} \leq 1 \forall j \in J, p \in P, d \in D \\
\sum_{t \in T_{d}}\left(Z_{j, p, t, d}^{S}-Z_{j, p, t, d}^{E}\right)=0 \forall j \in J, p \in P, d \in D \\
T_{j, p, d}^{S}=\sum_{t \in T_{d}} t \cdot Z_{j, p, t, d}^{S} \forall j \in J, p \in P, d \in D \\
T_{j, p, d}^{E}=\sum_{t \in T_{d}} t \cdot Z_{j, p, t, d}^{E} \forall j \in J, p \in P, d \in D \\
Z_{j, p, t} Z_{t^{\prime} \leq t}^{S}-\sum_{t^{\prime}<t} Z_{j, p, t^{\prime}, d}^{E} \forall j \in J, p \in P, t \in T_{d}, d \in D \\
N C_{i} X_{i, t}+\sum_{j \in J_{i}} Y_{i, j, t} \leq N C_{i} \forall i \in I, t \in T \\
Y_{i, j, t} \leq \sum_{p \in P} Z_{j, p, t} \forall i \in I, j \in J, t \in T \\
\sum_{p \in P} Z_{j, p, t} \leq 1 \forall j \in J, t \in T
\end{gathered}
$$

Flowrate constraints

$$
\begin{gathered}
F_{i}^{\min } X_{i, t} \leq F_{i, t} \leq F_{i}^{\max } X_{i, t} \forall i \in I, t \in T \\
F_{i, j}^{\min } Y_{i, j, t} \leq F_{i, j, t} \leq F_{i, j}^{\max } Y_{i, j, t} \quad \forall i \in I, j \in J, t \in T
\end{gathered}
$$




$$
F_{j}^{\min } Z_{j, p, t} \leq F_{j, p, t} \leq F_{j}^{\max } Z_{j, p, t} \forall i \in I, p \in P, t \in T
$$

Event point constraints

$$
\begin{array}{cl}
T_{j, e} \geq T_{j, p, d}^{S}-H_{d}\left(1-E_{j, p, e}\right) & \forall j \in J, p \in P, e \in E_{d}, d \in D \\
T_{j, e} \leq T_{j, p, d}^{S}+H_{d}\left(1-E_{j, p, e}\right) & \forall j \in J, p \in P, e \in E_{d}, d \in D \\
\sum_{e \in E_{d}} E_{j, p, e}=\sum_{t \in T_{d}} Z_{j, p, t, d}^{S} & \forall j \in J, p \in P, d \in D \\
T_{j, e} \geq T_{j, e-1} & \forall j \in J, e \in E \\
\sum_{p \in P} E_{j, p, e} \geq \sum_{p \in P} E_{j, p, e+1} & \forall j \in J, d \in D, e \in E_{d}^{L}
\end{array}
$$

Transition constraints

$$
\begin{array}{cc}
\sum_{p \in P} E_{j, p, e}+\sum_{p \in P} S_{j, p, e}=1 & \forall j \in J, e \in E \\
\sum_{n \in P} E_{j, n, e+1}+S_{j, p, e+1} \geq E_{j, p, e}+S_{j, p, e} & \forall j \in J, p \in P, e \in E-\{\mathrm{E}\} \\
W_{j, p, n, e} \geq E_{j, p, e}+S_{j, p, e}+E_{j, n, e+1}-1 \forall j \in J, p, n \in P, e \in E-\{\mathrm{E}\}, p \neq n \\
T_{j, p, d}^{E}=T_{j, p, d}^{S}+\left(\sum_{t \in T_{d}} Z_{j, p, t}-1\right), \quad \forall j \in J, p \in P, d \in D
\end{array}
$$

